\title{
Minimum Number of Colors: the Turk's Head Knots Case Study
}

\author{
Pedro Lopes ${ }^{1,2}$ \\ João Matias ${ }^{2}$ \\ 1 Center for Mathematical Analysis, Geometry and Dynamical Systems, Instituto Superior Técnico, University \\ of Lisbon, Portugal \\ 2 Department of Mathematics, Instituto Superior Técnico, University of Lisbon, Portugal
}

received $4^{\text {th }} \mathrm{Feb} .2012$, revised $18^{\text {th }}$ Jan. 2015, accepted $12^{\text {th }}$ June 2015.

An $r$-coloring of a knot diagram is an assignment of integers modulo $r$ to the arcs of the diagram such that at each crossing, twice the the number assigned to the over-arc equals the sum of the numbers assigned to the under-arcs, modulo $r$. The number of $r$-colorings is a knot invariant i.e., for each knot, it does not depend on the diagram we are using for counting them. In this article we calculate the number of $r$-colorings for the so-called Turk's Head Knots, for each modulus $r$. Furthermore, it is also known that whenever a knot admits an $r$-coloring using more than one color then all other diagrams of the same knot admit such $r$-colorings (called non-trivial $r$-colorings). This leads to the question of what is the minimum number of colors it takes to assemble such an $r$-coloring for the knot at issue. In this article we also estimate and sometimes calculate exactly what is the minimum numbers of colors for each of the Turk's Head Knots, for each relevant modulus $r$.

Keywords: Knots, Turk's head knots, colorings, colors, minimum number of colors

\section{Introduction}

Given an integer $r>1$ and a knot diagram, $D_{K}$, of a given knot $K$, a (Fox) $r$-coloring of $D_{K}(\operatorname{Fox}(1961))$ is an assignment of integers mod $r$ (i.e., numbers from $\mathbb{Z}_{r}=\{0,1,2, \ldots, r-1\} \bmod r$ ) to the arcs of the diagram such that, at each crossing of $D_{K}$, the equality "twice the color on the over-arc equals the sum of the colors on the under-arcs" holds $(\bmod r)$. The Fox $r$-colorings can be alternatively envisaged as follows. Assign a variable to each arc of $D_{K}$ and at each crossing read off the equation $2 y-x-z=0$ where $y$ is the variable assigned to the over-arc and $x$ and $z$ are the variables assigned to the under-arcs (Figure 1).

A system of linear homogeneous equations is thus associated to each knot diagram. The solutions of this system of equations mod $r$ constitute the $r$-colorings of $D_{K}$. There are always the trivial colorings i.e., the colorings where each arc is assigned the same color. Upon performing a Reidemeister move on $D_{K}$ endowed with an $r$-coloring, we can consistently assign colors to the arcs on the transformed portion of diagram in a unique way so that we obtain an $r$-coloring on the new diagram. Furthermore, if we undo this Reidemeister move, we can reassign colors so that we obtain the original $r$-coloring on $D_{K}$. There is thus a bijection between the $r$-colorings of two knot diagrams related by a finite number of Reidemeister 


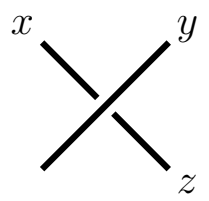

Fig. 1: In the context of knot colorings, arcs of a diagram stand for variables. At each crossing the equation "twice the over-arc equals the sum of the under-arcs" is read off.

moves (Lopes (2003)). Hence, the number of $r$-colorings is a knot invariant. Furthermore, this consistent assignment or reassignment of colors upon the performance of Reidemeister moves on colored diagrams takes trivial $r$-colorings to trivial $r$-colorings and non-trivial $r$-colorings to non-trivial $r$-colorings (nontrivial $r$-colorings being those that use at least two colors). In this way, the existence or not of non-trivial $r$-colorings is also a knot invariant.

Assume, then, a knot admits non-trivial $r$-colorings. What is the minimum number of colors needed to set up a non-trivial coloring over all diagrams of this knot?

Definition 1.1 Let $r$ be an integer greater than 1 . Let $K$ be a knot, $D_{K}$ one of its diagrams. Assume $K$ admits non-trivial $r$-colorings and let $n_{D_{K}, r}$ stand for the least number of colors it takes to set up a non-trivial $r$-coloring of $D_{K}$. We call

$$
\min \left\{n_{D_{K}, r} \mid D_{K} \text { is a diagram of } K\right\}
$$

the minimum number of colors of $K \bmod r$, and denote it $\operatorname{mincol}_{r}(K)$.

This is tautologically a knot invariant. Since to each knot there correspond infinitely many diagrams, the calculation of minimum number of colors by direct application of the definition is impossible. Nonetheless in this article we are able to make reasonable estimates and sometimes even calculate exactly the minimum number of colors of the knots at issue.

We remarked before that $r$-colorings of a knot $K$ can be regarded as the solutions of a system of linear homogeneous equations over $\mathbb{Z}_{r}$ read off the diagram of $K$ under study. Upon performance of Reidemeister moves on this diagram, the matrix of the coefficients of the linear homogeneous system of equations undergoes elementary transformations as described in Lickorish (1997) on page 50. In this way, the equivalence class of the matrix of the coefficients modulo elementary transformations is a knot invariant. Furthermore, due to the fact that any row vector (respect., column vector) is a linear combination of the other row vectors (respect., column vectors), it turns out that the absolute value of any first minor of this matrix yields the same value and is thus also a knot invariant. This first minor is called the determinant of the knot $K$, and is denoted det $K$. See also Lopes and Matias (2012) and Kauffman and Lopes (2009).

The topic of minimum number of colors was set forth in Harary and Kauffman (1999) where the Kauffman-Harary conjecture was presented. Given a prime $p$, this conjecture states that a non-trivial $p$-coloring on a reduced diagram of an alternating knot of prime determinant $p$, assigns different colors to different arcs. This conjecture has now been proven in Mattman and Solis (2009). 
There are other articles on minimum number of colors addressing the actual minimum for a given $r$ coloring. Satoh (Satoh (2009) ) shows that any non-trivial 5-coloring can be realized with 4 colors; Oshiro (Oshiro (2010)) shows that any 7-coloring can be realized with 4 colors. On the other hand, Saito (Saito (2010)) gives a condition for the minimum number of colors in a non-trivial $p$-coloring to be greater than 4 , for prime $p>7$. In Lopes and Matias (2012) it is conjectured that the minimum number of colors modulo $r$ of a given knot $K$ depends on the least common prime factor to $r$ and $\operatorname{det} K$. To support it, it is proved that this is true when this common prime factor is $2,3,5$, and 7 with the minima $2,3,4$, and 4 , respectively.

In Kauffman and Lopes (2008), the torus knots of type $(2, n)$, the $T(2, n)$ 's, were investigated. A formula for the number of $r$-colorings was established for each $n$, thereby allowing one to realize for which pairs $(r, n)$ there are non-trivial $r$-colorings of $T(2, n)$. For these cases, and relying on the features of modular arithmetic, estimates and sometimes actual minima were presented for the minimum number of colors. Furthermore, a sequence of transformations on the standard diagrams of the $T(2, n)$ 's were defined that helped on further decreasing the number of colors in infinitely many cases.

For each $n \in \mathbb{Z}^{+}$, the Turk's head knot on 3 strands, denoted $T H K(3, n)$, is the closure of the braid $\left(\sigma_{2} \sigma_{1}^{-1}\right)^{n} \in B_{3}$. Figure 2 illustrates $\operatorname{THK}(3,2)$ along with $\sigma_{1}, \sigma_{2}$, and their inverses.
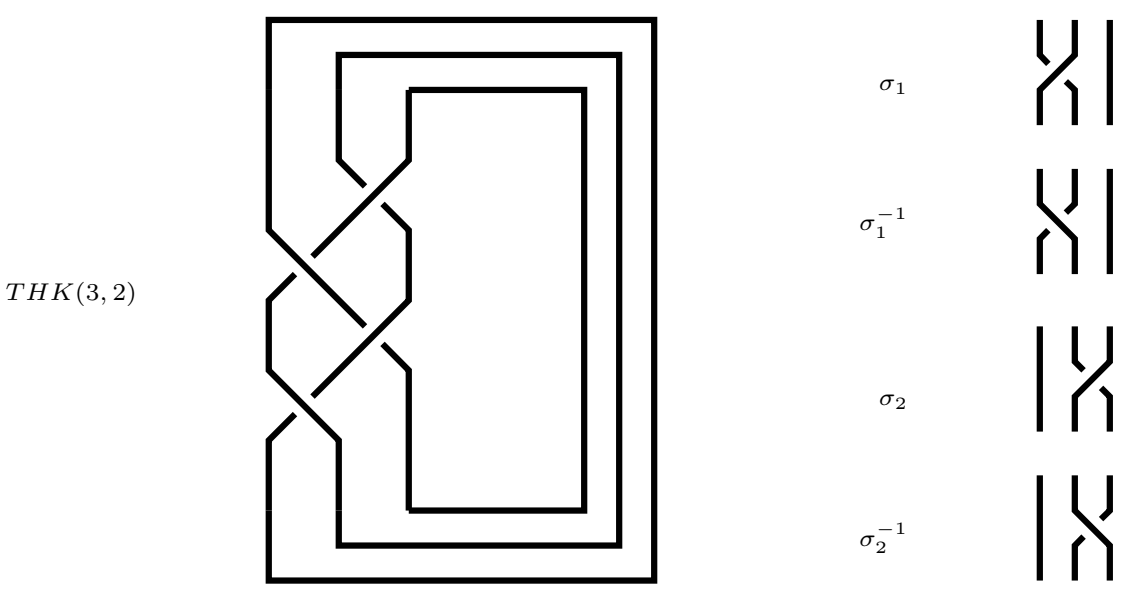

Fig. 2: On the left, $\operatorname{TH} K(3,2)$ as the closure of $\left(\sigma_{2} \sigma_{1}^{-1}\right)^{2} \in B_{3}$. On the right, from top to bottom: $\sigma_{1}, \sigma_{1}^{-1}, \sigma_{2}$, $\sigma_{2}^{-1}$.

See Birman (1974) for further information on braids. The knot diagrams of $T H K(3, n)$ obtained by the braid closure of $\left(\sigma_{2} \sigma_{1}^{-1}\right)^{n} \in B_{3}$, will be called standard diagrams of $T H K(3, n)$, in the sequel.

The present article is a study of the Turk's Head knots on three strands in the spirit of Kauffman and Lopes (2008). The fact that the $T H K(3, n)$ 's are more complex than the $T(2, n)$ 's turned this article into a harder project when compared to Kauffman and Lopes (2008). We remark in particular that the integration of a system of linear homogeneous equations over the modular integers falls outside the standard techniques of Linear Algebra, since in general the modular integers do not constitute a field. Nonetheless 
we are able to present a formula (see Theorem 1.1 for the number of $r$-colorings for the knot $T H K(3, n)$, for any positive integers $n$ and $r>1$. This corresponds to the integration of each system of linear homogeneous equations from an infinite family of such systems, over a generic ring of modular integers.

Here are the main results in this article. We remark that given two positive integers $a, b$, we let $\operatorname{gcd}(a, b)$ stand for their greatest common divisor. We further let $a \mid b$ stand for $a$ divides $b$ and $a \nmid b$ stand for $a$ does not divide $b$.

Theorem 1.1 Given positive integers $n$ and $r>1$, the number of $r$-colorings of $T H K(3, n)$, denoted $\# \operatorname{col}_{r}$ THK $(3, n)$, is

$$
\text { \# } \operatorname{col}_{r} \text { THK }(3, n)=\left\{\begin{array}{ll}
r \cdot\left(\operatorname{gcd}\left(u_{n-1}, r\right)\right)^{2} & \text { if } n \text { is odd } \\
r \cdot \operatorname{gcd}\left(5 u_{n-1}, r\right) \cdot \operatorname{gcd}\left(u_{n-1}, r\right) & \text { if } n \text { is even }
\end{array},\right.
$$

where $u_{n}$ is the solution of the linear recurrence problem with "initial values"

$$
-u_{n}+3 u_{n-2}-u_{n-4}=0, \quad u_{-3}=-1, \quad u_{-2}=-1, \quad u_{-1}=0, \quad u_{0}=1 .
$$

We remark that the solution of this problem is:

$$
u_{n}=\frac{1}{\sqrt{5}}\left[\left(\frac{1+\sqrt{5}}{2}\right)^{n+2}-\left(\frac{-1+\sqrt{5}}{2}\right)^{n}-\left(\frac{1-\sqrt{5}}{2}\right)^{n+2}+\left(\frac{-1-\sqrt{5}}{2}\right)^{n}\right] .
$$

\section{Corollary 1.1}

$$
0 \neq \operatorname{det} \operatorname{THK}(3, n)=\left\{\begin{array}{ll}
\left(u_{n-1}\right)^{2} & \text { if } n \text { is odd } \\
5\left(u_{n-1}\right)^{2} & \text { if } n \text { is even }
\end{array} .\right.
$$

In particular, the determinant of a knot classifies the knots in the THK(3,n) family. Furthermore, for any $k \in \mathbb{Z}^{+}, 5 \mid \operatorname{det} T H K(3,2 k)$ and $5 \nmid \operatorname{det} T H K(3,2 k+1)$.

Corollary 1.2 There are non-trivial r-colorings of $T H K(3, n)$ if, and only if,

- $\operatorname{gcd}\left(u_{n-1}, r\right)>1$

or

- $n$ is even and $5 \mid r$

Theorem 1.2 Let $n$ and $r$ be positive integers.

1 .

$$
2 \mid r \text { and } 3 \mid n \quad \text { if, and only if, } \quad \operatorname{mincol}_{r} T H K(3, n)=2 .
$$

2.

$$
(2 \nmid r \text { or } 3 \nmid n) \text { and } 3 \mid r \text { and } 4 \mid n \quad \text { if, and only if, } \quad \text { mincol }_{r} T H K(3, n)=3 \text {. }
$$


3.

$$
\begin{gathered}
(2 \nmid r \text { or } 3 \nmid n) \text { and }(3 \nmid r \text { or } 4 \nmid n) \text { and }[(5 \mid r \text { and } 2 \mid n) \text { or }(7 \mid r \text { and } 8 \mid n)] \\
\text { if, and only if, } \quad \operatorname{mincol}_{r} T H K(3, n)=4 .
\end{gathered}
$$

4. If

$$
\begin{gathered}
(2 \nmid r \text { or } 3 \nmid n) \text { and }(3 \nmid r \text { or } 4 \nmid n) \text { and }(5 \nmid r \text { or } 2 \nmid n) \text { and }(7 \nmid r \text { or } 8 \nmid n) \\
\text { and }(11 \mid r \text { and } 5 \mid n) \text { then } \text { mincol }_{r} T H K(3, n)=5 \text {. }
\end{gathered}
$$

It is easy to see that each statement in Theorem 1.2 gives rise to infinitely many knots for which the minimum number of colors modulo infinitely many $r$ 's is exactly determined. For example, according to the statement 4., for any positive integers $m$ and $l$, we have:

$$
\text { mincol }_{11 \cdot 13^{l}} \text { THK }\left(3,5 \cdot 17^{m}\right)=5 .
$$

We introduce the mapping $\psi$ which associates to each modulus $r$, the least positive integer $n$ such that $r \mid u_{n-1}$.

Definition 1.2 For any integer $r>1$ set

$$
\psi(r):=\min \left\{q \in \mathbb{Z}^{+}|r| u_{q-1}\right\} .
$$

When $r$ is a prime other than $5, \psi(r)$ is the least number of $\sigma_{2} \sigma_{1}^{-1}$ that should be juxtaposed in order to obtain a non trivial $r$-coloring, namely in $T H K(3, \psi(r))$. The first few values of the $\psi$ function are displayed in Table 2 right after the References to this article.

Theorem 1.3 Let p be a prime greater than 11.

1. Assume $\psi(p)$ is odd.

(a) If $5^{\frac{p-1}{2}} \equiv-1$, then mincol $_{p} T H K(3, \psi(p)) \leq \frac{p+1}{2}(\bmod p)$.

(b) If $5^{\frac{p-1}{2}} \equiv 1$, then $\operatorname{mincol}_{p} T H K(3, \psi(p)) \leq \frac{p-1}{2}(\bmod p)$.

2. Assume $\psi(p)$ is even.

(a) If $4 \mid \psi(p)$, then mincol $_{p} T H K(3, \psi(p)) \leq \psi(p)-1$.

(b) If $4 \nmid \psi(p)$, then mincol $_{p} T H K(3, \psi(p)) \leq \psi(p)-5$.

Definition 1.3 For any positive integers $n$ and $r$, such that $\operatorname{gcd}\left(u_{n-1}, r\right)>1$, set

$$
\left\langle u_{n-1}, r\right\rangle_{\psi}
$$

to be the least common prime factor (greater than 5 ) of $r$ and $u_{n-1}$, which minimizes $\psi$. 
Corollary 1.3 Let $r$ and $n$ be positive integers such that $\operatorname{gcd}\left(u_{n-1}, r\right)>1$. Set

$$
p:=\left\langle u_{n-1}, r\right\rangle_{\psi} .
$$

Then,

1. Assume $\psi(p)$ is odd.

(a) If $5^{\frac{p-1}{2}} \equiv-1$, then mincol $_{r} T H K(3, n) \leq \frac{p+1}{2}(\bmod p)$.

(b) If $5^{\frac{p-1}{2}} \equiv 1$, then mincol $_{r} T H K(3, n) \leq \frac{p-1}{2}(\bmod p)$.

2. Assume $\psi(p)$ is even.

(a) If $4 \mid \psi(p)$, then mincol $T H K(3, n) \leq \psi(p)-1$.

(b) If $4 \nmid \psi(p)$, then mincol $_{r} T H K(3, n) \leq \psi(p)-5$.

We establish below (Corollary 2.4 that, for prime $p \neq 5$, if $\psi(p)$ is odd then it is bounded above by $(p+1) / 2$, whereas if $\psi(p)$ is even, it is bounded above by $p+1$. Then, for the odd $p$ case, Theorem 1.3 provides good estimates, roughly half the number of colors available $(p)$. On the other hand, for the even $\psi(p)$ case, the estimates are coarser. The worst case is $\psi(p)=p+1$ where the results provide an estimate which equals the number of colors available (which is $p$ ).

We then ran a program in Mathematica to have an idea of how many times this $\psi(p)=p+1$ situation occurs over all primes $p$. Our program did this for the first 100, 000 primes in steps of 10,000. The results are displayed in Table 1 . This seems to indicate that around $40 \%$ of the primes, $p$, lead to $\psi(p)=p+1$.

\begin{tabular}{|c||c|c|c|c|c|c|c|c|c|c|}
\hline$N^{p}$ & 10,000 & 20,000 & 30,000 & 40,000 & 50,000 & 60,000 & 70,000 & 80,000 & 90,000 & 100,000 \\
\hline \hline$N_{\psi(p)}$ & 3,969 & 7910 & 11,853 & 15,760 & 19,738 & 23,661 & 27,589 & 31,499 & 35,404 & 39,343 \\
\hline \hline$N_{\psi(p)} / N^{p}$ & 0.3969 & 0.3955 & 0.3951 & 0.394 & 0.39476 & 0.39435 & 0.394129 & 0.393738 & 0.393378 & 0.39343 \\
\hline
\end{tabular}

Tab. 1: $N^{p}$ is the number of consecutive primes; $N_{\psi(p)}$ is the number of primes $p$ for which $\psi(p)=p+1$.

We ran another program to realize what is the percentage of distinct colors used over the total number of colors for the colorings we used in this situation. These colorings were induced by introducing either colors $0,1,0$ or colors $1,2,0$ on the top of the standard diagram of the $T H K(3, \psi(p))$ for each of these $p$ 's such that $\psi(p)=p+1$ and $p>7$. For the first 100, 000 such cases there was a minimum of $69.2308 \%$ and a maximum of $75.0004 \%$ of colors used.

In Section 2 we prove Theorems 1.1, 1.2, and 1.3, along with their corollaries. 


\section{Proofs}

In this Section we provide the proofs of the results stated in the Introduction.

\subsection{Proof of Theorem 1.1}

In this Subsection we prove Theorem 1.1. which yields a formula for the number of $r$-colorings of $T H K(3, n)$, along with its corollaries. For that, we start by studying the propagation of colors $a, b, c$ of an $r$-coloring, down $\left(\sigma_{2} \sigma_{1}^{-1}\right)^{n}$ (in Figure 3 the case $n=1$ is displayed). We recall that this means that at each crossing, the equation "twice the color on the over-arc equals the sum of colors on the under-arcs" is satisfied modulo $r$. At each crossing, we use this rule to write the color of the lower under-arc in terms of the color of over-arc and of the color of the upper under-arc.

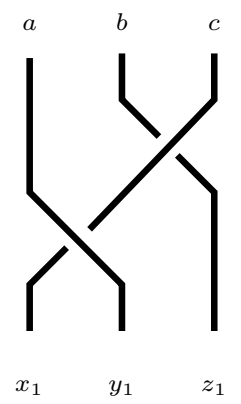

Fig. 3: Propagation of colors down $\sigma_{2} \sigma_{1}^{-1}$.

In Figure 3 we illustrate the propagation of colors $a, b, c$ down $\left(\sigma_{2} \sigma_{1}^{-1}\right)^{1}$, which, algebraically, translates into the system of equations 1

$$
\left\{\begin{array}{l}
x_{1}=2 a-c \\
y_{1}=a \\
z_{1}=2 c-b
\end{array} \quad \Longleftrightarrow\left[\begin{array}{l}
x_{1} \\
y_{1} \\
z_{1}
\end{array}\right]=\left[\begin{array}{ccc}
2 & 0 & -1 \\
1 & 0 & 0 \\
0 & -1 & 2
\end{array}\right]\left[\begin{array}{l}
a \\
b \\
c
\end{array}\right] .\right.
$$

In the sequel, we will use the following notation.

$$
C=\left[\begin{array}{ccc}
2 & 0 & -1 \\
1 & 0 & 0 \\
0 & -1 & 2
\end{array}\right]
$$

We thus start by setting $x_{0}=a, y_{0}=b, z_{0}=c$ at the top of the braid, from left to right; we call this the color input. The colors $x_{n}, y_{n}, z_{n}$ (from left to right) after $\left(\sigma_{2} \sigma_{1}^{-1}\right)^{n}$ are:

$$
\left[\begin{array}{l}
x_{n} \\
y_{n} \\
z_{n}
\end{array}\right]=C^{n}\left[\begin{array}{l}
a \\
b \\
c
\end{array}\right]
$$


In this way, each $r$-coloring of $T H K(3, n)$ will correspond to a solution of the system of linear equations, over the modulus $r$ :

$$
\left[\begin{array}{l}
a \\
b \\
c
\end{array}\right]=C^{n}\left[\begin{array}{l}
a \\
b \\
c
\end{array}\right]
$$

and vice-versa.

In the sequel we simplify the matrix $C^{n}$.

Proposition 2.1 Consider $T H K(3, n)$ given by the braid closure of $\left(\sigma_{2} \sigma_{1}^{-1}\right)^{n}$. Let $n$ be a non-negative integer. If the top strands of $\left(\sigma_{2} \sigma_{1}^{-1}\right)^{n}$ are endowed with colors $a, b$ and $c$ (from left to right) then the following hold:

(i) $x_{n}-y_{n}+z_{n}=a-b+c$

(ii) $x_{n}=4 x_{n-1}-4 x_{n-2}+x_{n-3}$;

$y_{n}=4 y_{n-1}-4 y_{n-2}+y_{n-3}$

$z_{n}=4 z_{n-1}-4 z_{n-2}+z_{n-3}$.

(iii) $x_{n}=3 x_{n-1}-x_{n-2}-x_{0}+y_{0}-z_{0}$;

$y_{n}=3 y_{n-1}-y_{n-2}-x_{0}+y_{0}-z_{0}$

$z_{n}=3 z_{n-1}-z_{n-2}-x_{0}+y_{0}-z_{0}$.

Proof: To prove $(i)$ we note that:

$$
\left[\begin{array}{lll}
1 & -1 & 1
\end{array}\right] C=\left[\begin{array}{lll}
1 & -1 & 1
\end{array}\right]\left[\begin{array}{ccc}
2 & 0 & -1 \\
1 & 0 & 0 \\
0 & -1 & 2
\end{array}\right]=\left[\begin{array}{lll}
1 & -1 & 1
\end{array}\right] .
$$

In this way, we obtain:

$$
\left[x_{n}-y_{n}+z_{n}\right]=\left[\begin{array}{lll}
1 & -1 & 1
\end{array}\right]\left[\begin{array}{l}
x_{n} \\
y_{n} \\
z_{n}
\end{array}\right]=\left[\begin{array}{lll}
1 & -1 & 1
\end{array}\right] C^{n}\left[\begin{array}{l}
a \\
b \\
c
\end{array}\right]=[a-b+c] .
$$

In order to prove $(i i)$ we note that, the characteristic polynomial of $C$ is

$$
\operatorname{det}\left(C-I_{3} x\right)=-x^{3}+4 x^{2}-4 x+1 .
$$

Via the Cayley-Hamilton Theorem,

which amounts to

$$
\left(-C^{3}+4 C^{2}-4 C+I_{3}\right)\left[\begin{array}{l}
x_{n} \\
y_{n} \\
z_{n}
\end{array}\right]=0
$$

$$
-\left[\begin{array}{l}
x_{n+3} \\
y_{n+3} \\
z_{n+3}
\end{array}\right]+4\left[\begin{array}{l}
x_{n+2} \\
y_{n+2} \\
z_{n+2}
\end{array}\right]-4\left[\begin{array}{l}
x_{n+1} \\
y_{n+1} \\
z_{n+1}
\end{array}\right]+\left[\begin{array}{l}
x_{n} \\
y_{n} \\
z_{n}
\end{array}\right]=0
$$


Thus yielding the indicated recurrence relations for $x_{n}, y_{n}, z_{n}$.

To prove (iii) we start by using:

$$
\begin{aligned}
{\left[\begin{array}{l}
x_{n} \\
y_{n} \\
z_{n}
\end{array}\right]=4\left[\begin{array}{l}
x_{n-1} \\
y_{n-1} \\
z_{n-1}
\end{array}\right]-4\left[\begin{array}{l}
x_{n-2} \\
y_{n-2} \\
z_{n-2}
\end{array}\right]+\left[\begin{array}{l}
x_{n-3} \\
y_{n-3} \\
z_{n-3}
\end{array}\right]=} \\
\left.=3\left[\begin{array}{l}
x_{n-1} \\
y_{n-1} \\
z_{n-1}
\end{array}\right]-\left[\begin{array}{l}
x_{n-2} \\
y_{n-2} \\
z_{n-2}
\end{array}\right]+\left[\begin{array}{ccc}
2 & 0 & -1 \\
1 & 0 & 0 \\
0 & -1 & 2
\end{array}\right]-3\left[\begin{array}{ccc}
2 & 0 & -1 \\
1 & 0 & 0 \\
0 & -1 & 2
\end{array}\right]+I_{3}\right)\left[\begin{array}{l}
x_{n-3} \\
y_{n-3} \\
z_{n-3}
\end{array}\right]= \\
=3\left[\begin{array}{l}
x_{n-1} \\
y_{n-1} \\
z_{n-1}
\end{array}\right]-\left[\begin{array}{l}
x_{n-2} \\
y_{n-2} \\
z_{n-2}
\end{array}\right]+\left[\begin{array}{lll}
-1 & 1 & -1 \\
-1 & 1 & -1 \\
-1 & 1 & -1
\end{array}\right]\left[\begin{array}{l}
x_{n-3} \\
y_{n-3} \\
z_{n-3}
\end{array}\right]= \\
=3\left[\begin{array}{l}
x_{n-1} \\
y_{n-1} \\
z_{n-1}
\end{array}\right]-\left[\begin{array}{l}
x_{n-2} \\
y_{n-2} \\
z_{n-2}
\end{array}\right]+\left[\begin{array}{l}
-x_{0}+y_{0}-z_{0} \\
-x_{0}+y_{0}-z_{0} \\
-x_{0}+y_{0}-z_{0}
\end{array}\right]
\end{aligned}
$$

where in the last equality $(i)$ was used. This concludes the proof of Proposition 2.1 .

Noting that $C$ is invertible, the preceding recurrence relations allows us to define $x_{n}, y_{n}, z_{n}$ for negative values with the relation,

$$
x_{n-3}=4 x_{n-2}-4 x_{n-1}+x_{n},
$$

and analogously for $y_{n}$ and $z_{n}$. We may, thus, define, for any $n \in \mathbb{Z}$,

$$
\left[\begin{array}{l}
x_{n} \\
y_{n} \\
z_{n}
\end{array}\right]=C^{n}\left[\begin{array}{l}
a \\
b \\
c
\end{array}\right]=\left[\begin{array}{ccc}
2 & 0 & -1 \\
1 & 0 & 0 \\
0 & -1 & 2
\end{array}\right]^{n}\left[\begin{array}{l}
a \\
b \\
c
\end{array}\right]
$$

We remark that the coefficients of $a, b, c$ in the leftmost color (as well as in the middle and in the rightmost colors), satisfy a recurrence relation similar to the one $x_{n}$ does.

Definition 2.1 Set:

$$
\begin{aligned}
& x_{n}=a_{n} a+b_{n} b+c_{n} c, \\
& y_{n}=a_{n}^{\prime} a+b_{n}^{\prime} b+c_{n}^{\prime} c, \\
& z_{n}=a_{n}^{\prime \prime} a+b_{n}^{\prime \prime} b+c_{n}^{\prime \prime} c .
\end{aligned}
$$

We have:

$$
\left[\begin{array}{l}
x_{n} \\
y_{n} \\
z_{n}
\end{array}\right]=C^{n}\left[\begin{array}{l}
a \\
b \\
c
\end{array}\right]=\left[\begin{array}{ccc}
a_{n} & b_{n} & c_{n} \\
a_{n}^{\prime} & b_{n}^{\prime} & c_{n}^{\prime} \\
a_{n}^{\prime \prime} & b_{n}^{\prime \prime} & c_{n}^{\prime \prime}
\end{array}\right]\left[\begin{array}{l}
a \\
b \\
c
\end{array}\right] .
$$

\section{Corollary 2.1 Keeping the conditions of the proposition above:}

(i) $a_{n}=4 a_{n-1}-4 a_{n-2}+a_{n-3}$;

$b_{n}=4 b_{n-1}-4 b_{n-2}+b_{n-3}$

$c_{n}=4 c_{n-1}-4 c_{n-2}+c_{n-3}$. 
(ii) $\begin{aligned} a_{n} & =3 a_{n-1}-a_{n-2}-1 \\ b_{n} & =3 b_{n-1}-b_{n-2}+1 \\ c_{n} & =3 c_{n-1}-c_{n-2}-1\end{aligned}$

Proof: We will prove the proposition for the sequence $a_{n}$, since the other proofs are analogous. Consider the matrix $C^{n}$. Then, one realizes $a_{n}$ is the leftmost color after $\left(\sigma_{2} \sigma_{1}^{-1}\right)^{n}$, when $x_{0}=1, y_{0}=0, z_{0}=0$ i.e., $x_{n}=a_{n}$ when $x_{0}=1, y_{0}=0, z_{0}=0$. Applying Proposition 2.1. we obtain the desired relations.

More generally, the entries of $C^{n}$ satisfy a recurrence relation similar to the one $x_{n}, y_{n}$, and $z_{n}$ do, as $c_{i j}^{n}$, the entry $i j$ of $C^{n}$, can be interpreted as the colors of the $i-t h$ strand with the top colors equal to zero except for the $j-t h$ strand that takes 1 as initial color.

Before proceeding to the next result, let us look at the first few powers of $C$ :

$$
C=\left[\begin{array}{ccc}
2 & 0 & -1 \\
1 & 0 & 0 \\
0 & -1 & 2
\end{array}\right], \quad C^{2}=\left[\begin{array}{ccc}
4 & 1 & -4 \\
2 & 0 & -1 \\
-1 & -2 & 4
\end{array}\right], \quad C^{3}=\left[\begin{array}{ccc}
9 & 4 & -12 \\
4 & 1 & -4 \\
-4 & -4 & 9
\end{array}\right] .
$$

Corollary 2.2 The powers of the matrix C satisfy:

$$
C^{n}=\left[\begin{array}{ccc}
a_{n} & b_{n} & -b_{n+1} \\
a_{n-1} & b_{n-1} & -b_{n} \\
-b_{n} & -a_{n-1} & a_{n}
\end{array}\right], n \in \mathbb{Z}
$$

Proof: We already saw that the entries of the matrices $C^{n}$ satisfy a specific recurrence relation, which is similar to the ones $a_{n}$, and $b_{n}$ do. So, it is enough to verify that the first 3 powers of $C$ satisfy the expression above, and to use induction to establish the result. We leave the details to the reader.

We recall we want to establish a formula which yields the number of $r$-colorings of $T H K(3, n)$. In order to do that, we solve the following system of linear equations over $\mathbb{Z}_{r}$ :

$$
C^{n}\left[\begin{array}{l}
x_{0} \\
y_{0} \\
z_{0}
\end{array}\right]=\left[\begin{array}{l}
x_{0} \\
y_{0} \\
z_{0}
\end{array}\right]
$$

which, upon rewriting and applying Corollary 2.2 yields the following system of linear homogeneous equations over $\mathbb{Z}_{r}$ :

$$
\left[\begin{array}{ccc}
a_{n}-1 & b_{n} & -b_{n+1} \\
a_{n-1} & b_{n-1}-1 & -b_{n} \\
-b_{n} & -a_{n-1} & a_{n}-1
\end{array}\right]\left[\begin{array}{l}
x_{0} \\
y_{0} \\
z_{0}
\end{array}\right]=\left[\begin{array}{l}
0 \\
0 \\
0
\end{array}\right]
$$

The coefficient matrix in (4) can be further simplified as we will now show.

Corollary 2.3 For any positive integer $n$ :

$$
\begin{aligned}
a_{n}-a_{n-1}-b_{n} & =1, \\
b_{n}-b_{n-1}-a_{n-1} & =-1 .
\end{aligned}
$$


Proof: This follows from Corollary 2.2. We remark that $a_{n}, a_{n-1}$ and $-b_{n}$ are the bottom colors, from left to right, respectively, of the braid $\left(\sigma_{2} \sigma_{1}^{-1}\right)^{n}$ when $x_{0}=1, y_{0}=0$ and $z_{0}=0$. Therefore, by Proposition 2.1. we have:

$$
a_{n}-a_{n-1}+\left(-b_{n}\right)=1-0+0=1 .
$$

Also, $b_{n}, b_{n-1}$ and $-a_{n-1}$ are the bottom colors, from left to right, respectively, of the strings of the braid $\left(\sigma_{2} \sigma_{1}^{-1}\right)^{m}$ when $x_{0}=0, y_{0}=1$ and $z_{0}=0$. Again by Proposition 2.1

$$
b_{n}-b_{n-1}+\left(-a_{n-1}\right)=0-1+0=-1 .
$$

Now, by Corollary 2.3 we may conclude that:

$$
\left[\begin{array}{lll}
1 & -1 & 1
\end{array}\right]\left[\begin{array}{ccc}
a_{n} & b_{n} & -b_{n+1} \\
a_{n-1} & b_{n-1} & -b_{n} \\
-b_{n} & -a_{n-1} & a_{n}
\end{array}\right]-\left[\begin{array}{ccc}
1 & -1 & 1
\end{array}\right]=\left[\begin{array}{lll}
0 & 0 & 0
\end{array}\right] .
$$

In this way, by adding the first line to and subtracting the second line from the third line in the square matrix in (4), we obtain:

$$
\left[\begin{array}{ccc}
1 & 0 & 0 \\
0 & 1 & 0 \\
1 & -1 & 1
\end{array}\right]\left[\begin{array}{ccc}
a_{n}-1 & b_{n} & -b_{n+1} \\
a_{n-1} & b_{n-1}-1 & -b_{n} \\
-b_{n} & -a_{n-1} & a_{n}-1
\end{array}\right]=\left[\begin{array}{ccc}
a_{n}-1 & b_{n} & -b_{n+1} \\
a_{n-1} & b_{n-1}-1 & -b_{n} \\
0 & 0 & 0
\end{array}\right]
$$

We now define the sequences $u_{n}$ and $v_{n}$ which will relate to $a_{n}$ and $b_{n}$.

Definition 2.2 Let $u_{n}$ and $v_{n}$ be sequences defined recursively as follows:

$$
u_{-3}=-1, \quad u_{-2}=-1, \quad u_{-1}=0, \quad u_{0}=1, \quad \text { and } \text { if } n \geq 1 \quad u_{n}=3 u_{n-2}-u_{n-4},
$$

and,

$$
v_{-3}=7, \quad v_{-2}=2, \quad v_{-1}=3, \quad v_{0}=1, \quad \text { and if } n \geq 1 \quad v_{n}=3 v_{n-2}-v_{n-4} .
$$

Proposition 2.2 For $n \in \mathbb{N}$ we have:

$$
\begin{aligned}
a_{n} & =u_{n} v_{n}, & b_{n} & =u_{n-2} u_{n-1}, \\
a_{n}-1 & =u_{n-1} v_{n+1}, & b_{n}-1 & =u_{n} u_{n-3} .
\end{aligned}
$$

Proof: First we need to verify the cases $n=0,1,2,3,4$. We leave this task to the reader. We will prove by induction that $a_{n}=u_{n} v_{n}$ and $\left(a_{n}-1\right)=u_{n-1} v_{n+1}$. For $n \geq 0$ we assume the validity of the hypothesis for $n, n+1, n+2, n+3$ and $n+4$ and conclude that it is also valid for $n+5$. Proposition 2.1 will be used throughout the following calculations. 


$$
\begin{aligned}
u_{n+5} v_{n+5}= & \left(3 u_{n+3}-u_{n+1}\right)\left(3 v_{n+3}-v_{n+1}\right)= \\
= & 9 u_{n+3} v_{n+3}-3\left(3 u_{n+1}-u_{n-1}\right) v_{n+1}-3 u_{n+1} v_{n+3}+u_{n+1} v_{n+1}= \\
= & 9 a_{n+3}-9 a_{n+1}+3\left(a_{n}-1\right)-3\left(a_{n+2}-1\right)+a_{n+1}= \\
= & 9 a_{n+3}-15 a_{n+2}+4 a_{n+1}+3\left(4 a_{n+2}-4_{n+1}+a_{n}\right)= \\
= & -4 a_{n+3}+a_{n+2}+4\left(4 a_{n+3}-4 a_{n+2}+a_{n+1}\right)= \\
= & 4 a_{n+4}-4 a_{n+3}+a_{n+2}=a_{n+5} . \\
u_{n+4} v_{n+6} & =\left(3 u_{n+2}-u_{n}\right)\left(3 v_{n+4}-v_{n+2}\right)= \\
& =9 u_{n+2} v_{n+4}-3 u_{n}\left(3 v_{n+2}-v_{n}\right)-3 u_{n+2} v_{n+2}+u_{n} v_{n+2}= \\
& =9\left(a_{n+3}-1\right)-9\left(a_{n+1}-1\right)+3 a_{n}-3 a_{n+2}+\left(a_{n+1}-1\right)= \\
& =9 a_{n+3}-15 a_{n+2}+4 a_{n+1}-1+3\left(4 a_{n+2}-4 a_{n+1}+a_{n}\right)= \\
& =-4 a_{n+3}+a_{n+2}-1+4\left(4 a_{n+3}-4 a_{n+2}+a_{n+1}\right)= \\
& =4 a_{n+4}-4 a_{n+3}+a_{n+2}-1=a_{n+5}-1 .
\end{aligned}
$$

This ends the first part of the proof. Now, let us prove by induction that $b_{n}=u_{n-2} u_{n-1}$ and $\left(b_{n}-1\right)=$ $u_{n} u_{n-3}$. We assume again for $n \geq 0$ the validity of the hypothesis for $n, n+1, n+2, n+3$ and $n+4$, concluding that it is valid for $n+5$.

$$
\begin{aligned}
u_{n+3} u_{n+4}= & u_{n+3}\left(3 u_{n+2}-u_{n}\right)=3 b_{n+4}-\left(b_{n+3}-1\right)= \\
= & 3 b_{n+4}-b_{n+3}+1=b_{n+5} . \\
u_{n+5} u_{n+2} & =\left(3 u_{n+3}-u_{n+1}\right) u_{n+2}=3 b_{n+4}-b_{n+3}= \\
& =\left(3 b_{n+4}-b_{n+3}+1\right)-1=b_{n+5}-1 .
\end{aligned}
$$

This ends the proof.

The linear homogeneous system of equations over $\mathbb{Z}_{r}$ is now equivalent to:

$$
u_{n-1}\left[\begin{array}{ccc}
v_{n+1} & u_{n-2} & -u_{n} \\
v_{n-1} & u_{n-4} & -u_{n-2} \\
0 & 0 & 0
\end{array}\right]\left[\begin{array}{l}
x_{0} \\
y_{0} \\
z_{0}
\end{array}\right]=\left[\begin{array}{l}
0 \\
0 \\
0
\end{array}\right]
$$

By performing elementary operations on the lines of the matrix we obtain an even simpler coefficient 
matrix:

$$
\begin{aligned}
& {\left[\begin{array}{ccc}
v_{k+1} & u_{k-2} & -u_{k} \\
v_{k-1} & u_{k-4} & -u_{k-2} \\
0 & 0 & 0
\end{array}\right] \longrightarrow\left[\begin{array}{ccc}
v_{k+1}-2 v_{k-1} & u_{k-2}-2 u_{k-4} & -u_{k}+2 u_{k-2} \\
v_{k-1} & u_{k-4} & -u_{k-2} \\
0 & 0 & 0
\end{array}\right] \rightarrow } \\
& \longrightarrow\left[\begin{array}{ccc}
v_{k+1}-2 v_{k-1} & u_{k-2}-2 u_{k-4} & -u_{k}+2 u_{k-2} \\
-v_{k+1}+3 v_{k-1} & -u_{k-2}+3 u_{k-4} & u_{k}-3 u_{k-2} \\
0 & 0 & 0
\end{array}\right]= \\
&= {\left[\begin{array}{ccc}
v_{k-1}-v_{k-3} & u_{k-4}-u_{k-6} & -u_{k-2}+u_{k-4} \\
v_{k-3} & u_{k-6} & -u_{k-4} \\
0 & 0 & 0
\end{array}\right] \longrightarrow\left[\begin{array}{ccc}
v_{k-1} & u_{k-4} & -u_{k-2} \\
v_{k-3} & u_{k-6} & -u_{k-4} \\
0 & 0 & 0
\end{array}\right] . }
\end{aligned}
$$

Therefore, for $n$ odd, the coefficient matrix in (5) simplifies to:

$$
u_{n-1}\left[\begin{array}{ccc}
v_{2} & u_{-1} & -u_{1} \\
v_{0} & u_{-3} & -u_{-1} \\
0 & 0 & 0
\end{array}\right]=u_{n-1}\left[\begin{array}{ccc}
1 & 0 & -1 \\
1 & -1 & 0 \\
0 & 0 & 0
\end{array}\right] \longrightarrow u_{n-1}\left[\begin{array}{ccc}
1 & 0 & -1 \\
0 & -1 & 1 \\
0 & 0 & 0
\end{array}\right],
$$

whereas, for $n$ even, it simplifies to:

$$
u_{n-1}\left[\begin{array}{ccc}
v_{3} & u_{0} & -u_{2} \\
v_{1} & u_{-2} & -u_{0} \\
0 & 0 & 0
\end{array}\right]=u_{n-1}\left[\begin{array}{ccc}
3 & 1 & -4 \\
2 & -1 & -1 \\
0 & 0 & 0
\end{array}\right] \longrightarrow u_{n-1}\left[\begin{array}{ccc}
1 & 2 & -3 \\
0 & -5 & 5 \\
0 & 0 & 0
\end{array}\right]
$$

We can now establish the formulas for the number of the colorings in terms of $r$ and $u_{n-1}$ in Theorem 1.1. We state that part here again as Proposition 2.3 below for the reader's convenience. We recall we let $\operatorname{gcd}(a, b)$ stand for the greatest common divisor of the positive integers $a$ and $b$.

Proposition 2.3 Given positive integers $n$ and $r>1$,

$$
\text { \# } \operatorname{col}_{r} \text { THK }(3, n)=\left\{\begin{array}{ll}
r \cdot\left(\operatorname{gcd}\left(u_{n-1}, r\right)\right)^{2} & \text { if } n \text { is odd } \\
r \cdot \operatorname{gcd}\left(5 u_{n-1}, r\right) \cdot \operatorname{gcd}\left(u_{n-1}, r\right) & \text { if } n \text { is even }
\end{array} .\right.
$$

Proof: Let us suppose that $n$ is odd. The color input $a, b, c$ in $\left(\sigma_{2} \sigma_{1}^{-1}\right)^{n}$ induces an $r$-coloring in $T H K(3, n)$ if and only if:

$$
u_{n-1}\left[\begin{array}{ccc}
1 & 0 & -1 \\
0 & -1 & 1 \\
0 & 0 & 0
\end{array}\right]\left[\begin{array}{l}
a \\
b \\
c
\end{array}\right]={ }_{r}\left[\begin{array}{l}
0 \\
0 \\
0
\end{array}\right]
$$

We now count the solutions of this linear homogeneous system of equations over $\mathbb{Z}_{r}$ (see Kauffman and Lopes (2008), Claims 2.1 and 2.2). The third equation does not impose any restrictions on $a, b$, or $c$. 
The second equation, $u_{n-1}(-b+c)={ }_{r} 0$, yields $b-c=k \frac{r}{\operatorname{gcd}\left(u_{n-1}, r\right)}$ for $k=0,1, \ldots, \operatorname{gcd}\left(u_{n-1}, r\right)-1$. Note that for each positive integer $a$,

$$
a x={ }_{r} 0 \Leftrightarrow \operatorname{gcd}(a, r) x={ }_{r} 0 .
$$

Moreover, from the first equation, $a=c+k \frac{r}{\operatorname{gcd}\left(u_{n-1}, r\right)}$. So, both $b$ and $a$ have $\left(u_{n-1}, r\right)$ possibilities, compatible with each of the $r$ possibilities for $c$. We conclude that the indicated system of equations has $r \operatorname{gcd}\left(u_{n-1}, r\right)^{2}$ solutions over $\mathbb{Z}_{r}$.

Let us now suppose that $n$ is even. The color input $a, b, c$ in $\left(\sigma_{2} \sigma_{1}^{-1}\right)^{n}$ induces an $r$-coloring in $\operatorname{THK}(3, n)$ if and only if:

$$
u_{n-1}\left[\begin{array}{ccc}
1 & 2 & -3 \\
0 & -5 & 5 \\
0 & 0 & 0
\end{array}\right]\left[\begin{array}{l}
a \\
b \\
c
\end{array}\right] \equiv_{r}\left[\begin{array}{l}
0 \\
0 \\
0
\end{array}\right]
$$

Arguing as above, we conclude that the number of solutions over $\mathbb{Z}_{r}$ of the indicated system of equations is $r \cdot \operatorname{gcd}\left(5 u_{n-1}, r\right) \cdot \operatorname{gcd}\left(u_{n-1}, r\right)$, for even $n$. This concludes the proof.

Proposition 2.4 For $n \in \mathbb{Z}$,

$$
u_{n}=\frac{1}{\sqrt{5}}\left(\left(\frac{1+\sqrt{5}}{2}\right)^{n+2}-\left(\frac{-1+\sqrt{5}}{2}\right)^{n}-\left(\frac{1-\sqrt{5}}{2}\right)^{n+2}+\left(\frac{-1-\sqrt{5}}{2}\right)^{n}\right) \text {. }
$$

Proof: The sequence $u_{n}$ is the solution of the linear difference equation with the "initial values":

$$
-u_{n}+3 u_{n-2}-u_{n-4}=0, \quad u_{-3}=-1, \quad u_{-2}=-1, \quad u_{-1}=0, \quad u_{0}=1 .
$$

The general method of solving this type of initial value problem can be found in Henrici (1964), for instance. We leave the details to the reader.

This concludes the proof of Theorem 1.1 and of Corollary 1.2 .

The formulas for the determinants in Corollary 1.1 follow from the observation of the matrices 6 for the odd $n$ case and (7) for the even $n$ case; the fact that these determinants are always greater than zero follows from proving by induction that

$$
u_{n}>0 \quad \text { and } \quad u_{n}-u_{n-2}>0 \quad \text { for all } n>2 .
$$

\subsection{Proof of Theorem 1.2}




\subsubsection{Preliminaries}

We first establish Propositions 2.5, 2.6, 2.7 for they will be useful in the sequel.

Proposition 2.5 Suppose a knot admits a non-trivial s-coloring and $s \mid r$. Then this knot also admits a non-trivial $r$-coloring.

Proof: Since $s \mid r$, then the set

$$
\left\{0, \frac{r}{s}, 2 \frac{r}{s}, \ldots,(s-1) \frac{r}{s}\right\} \quad \bmod r
$$

is closed with respect to the $a * b:=2 b-a \bmod r$ operation. Moreover, the mapping

$$
\begin{aligned}
f: \mathbb{Z}_{s} \longrightarrow \mathbb{Z}_{r} \\
i \longrightarrow i \frac{r}{s}
\end{aligned}
$$

is injective and preserves the $*$ operation. In this way, if $\left(i_{1}, i_{2}, \ldots, i_{N}\right)$ is the sequence of colors mod $s$ one should assign to the sequence of arcs in a knot diagram of the knot under study to obtain a non-trivial $s$-coloring, then the sequence $\left(i_{1} \frac{r}{s}, i_{2} \frac{r}{s}, \ldots, i_{N} \frac{r}{s}\right)$ assigned to the same sequence of arcs, represents a non-trivial $r$-coloring of the same knot.

Proposition 2.6 Consider the positive integers $c, k, n$, and integer $r>1$. Suppose the standard diagram of THK $(3, n)$ admits a non-trivial $r$-coloring with c colors. Then the standard diagram of $T H K(3, k n)$ also admits a non-trivial $r$-coloring with c colors.

Proof: If the standard diagram of $T H K(3, n)$ admits a non-trivial $r$-coloring with $c$ colors, we consider this coloring in $\left(\sigma_{2} \sigma_{1}^{-1}\right)^{n}$ i.e., before braid closure. In this way, the sequence of colors on the top arcs (from left to right) equals the sequence of colors on the bottom arcs (from left to right). We then juxtapose $k$ copies of this colored $\left(\sigma_{2} \sigma_{1}^{-1}\right)^{n}$. Upon taking its closure we obtain a non-trivial $r$-coloring of $\operatorname{THK}(3, k n)$.

Definition 2.3 A knot is said split if there exist two disjoint open balls in 3-space, say $N_{1}$ and $N_{2}$, such that the knot is deformable into a knot, say $K$, such that

$$
K \subset N_{1} \cup N_{2}, \quad K \cap N_{1} \neq \emptyset, \quad K \cap N_{2} \neq \emptyset .
$$

Otherwise, the knot is said non-split.

Proposition 2.7 For any positive integer $n$, $T H K(3, n)$ is non-split.

Proof: Fix a positive integer $n$. If $T H K(3, n)$ were split, then for any integer $r>1$, there would be at least $r^{2} r$-colorings. Each of these $r^{2} r$-colorings stands for the $r$-coloring which results from trivially 
$r$-coloring each of the two open balls the knot splits into. But, setting $r$ equal to a prime larger than both $u_{n-1}$ and 5 , yields

$$
\# \operatorname{col}_{r} \text { THK(3,n) }=\left\{\begin{array}{ll}
r \cdot\left(\operatorname{gcd}\left(u_{n-1}, r\right)\right)^{2} & \text { if } n \text { is odd } \\
r \cdot\left(\operatorname{gcd}\left(5 u_{n-1}, r\right)\right) \cdot\left(\operatorname{gcd}\left(u_{n-1}, r\right)\right) & \text { if } n \text { is even }
\end{array}=r<r^{2} .\right.
$$

Hence, $\operatorname{TH} K(3, n)$ is non-split.

\subsubsection{Further analysis of the $u_{n}$ sequence and the $\psi$ mapping: towards the proof of Theorem 1.2}

In order to prove the "if" parts in Theorem 1.2, and to prove Theorem 1.3 , we analyze further the sequence $u_{n}$.

Proposition 2.8 For $n \in \mathbb{Z}$ we have:

$$
\begin{aligned}
u_{2 n} & =u_{2 n+1}+u_{2 n-1}, \\
u_{2 n+1} & =\frac{u_{2 n+2}+u_{2 n}}{5} .
\end{aligned}
$$

Proof: We only prove the inductive step:

$$
\begin{gathered}
u_{2 n+3}+u_{2 n+1}=3 u_{2 n+1}-u_{2 n-1}+u_{2 n+1}=5 u_{2 n+1}-u_{2 n+1}-u_{2 n-1}= \\
=u_{2 n+2}+\left(u_{2 n}-u_{2 n+1}-u_{2 n-1}\right)=u_{2 n+2}+0 . \\
\begin{aligned}
& u_{2 n+4}+u_{2 n+2}=3 u_{2 n+2}- u_{2 n}+u_{2 n+2}=5 u_{2 n+2}-u_{2 n+2}-u_{2 n}= \\
&=5\left(u_{2 n+3}+u_{2 n+1}\right)-u_{2 n+2}-u_{2 n}=5 u_{2 n+3}+\left(5 u_{2 n+1}-u_{2 n+2}-u_{2 n}\right)=5 u_{2 n+3}+0 .
\end{aligned}
\end{gathered}
$$

Proposition 2.9 (i) If $m$ is even, or $n$ is odd, then:

$$
u_{m+n}=u_{m+1} u_{n}-u_{m-1} u_{n-2} .
$$

(ii) If $m$ is even and $n$ is odd, then:

$$
u_{m+n}=u_{m} u_{n}-u_{m-1} u_{n-1} .
$$

Proof: To prove $(i)$ let us first suppose $m$ is an arbitrary integer, we have:

$$
\begin{aligned}
& u_{m+1}=u_{m+1} \times 1-u_{m-1} \times 0=u_{m+1} u_{1}-u_{m-1} u_{-1}, \\
& u_{m+3}=u_{m+1} \times 3-u_{m-1} \times 1=u_{m+1} u_{3}-u_{m-1} u_{1} .
\end{aligned}
$$


Also, if $m$ is even, by Proposition 2.8, we have:

$$
\begin{aligned}
& u_{m}=u_{m+1} \times 1-u_{m-1} \times(-1)=u_{m+1} u_{0}-u_{m-1} u_{-2}, \\
& u_{m+2}=u_{m+3}+u_{m+1}=u_{m+1} \times 4-u_{m-1} \times 1=u_{m+1} u_{2}-u_{m-1} u_{0} .
\end{aligned}
$$

Now, fixed $m$, we will do induction on $n$. Let us suppose that the equation 9 is true for a given $m$ and $n=k, k+2$, then:

$$
\begin{aligned}
u_{m+(k+4)} & =3 u_{m+(k+2)}-u_{m+k}=u_{m+1}\left(3 u_{k+2}-u_{k}\right)-u_{m-1}\left(3 u_{k}-u_{k-2}\right)= \\
& =u_{m+1} u_{k+4}-u_{m-1} u_{k+2}, \\
u_{m+(k-2)} & =3 u_{m+k}-u_{m+(k+2)}=u_{m+1}\left(3 u_{k}-u_{k+2}\right)-u_{m-1}\left(3 u_{k-2}-u_{k}\right)= \\
& =u_{m+1} u_{k-2}-u_{m-1} u_{k-4} .
\end{aligned}
$$

So, equation (9) is also verified for $n=k-2, k+4$. From here we may conclude that when $m$ is even equation (9) is true for every $n$, and when $m$ is odd equation (9) is true for $n$ odd.

To prove (ii) we use (i), and Proposition 2.8.

$$
\begin{aligned}
u_{m+n} & =u_{m+1} u_{n}-u_{m-1} u_{n-2}=\left(u_{m}-u_{m-1}\right) u_{n}-u_{m-1} u_{n-2} \\
& =u_{m} u_{n}-u_{m-1}\left(u_{n}+u_{n-2}\right)=u_{m} u_{n}-u_{m-1} u_{n-1} .
\end{aligned}
$$

We repeat here the definition of the $\psi$ mapping for the reader's convenience.

Definition 2.4 For any integer $r>1$ set

$$
\psi(r):=\min \left\{q \in \mathbb{Z}^{+}|r| u_{q-1}\right\}
$$

Proposition 2.10 $\psi$ is well-defined.

Proof: We will prove that, for any integer $r>1,\left\{q \in \mathbb{Z}^{+}|r| u_{q-1}\right\} \neq \emptyset$. Since it is a subset of $\mathbb{Z}^{+}$, then it has a minimum.

Fix an integer $r>1$ and set $U_{n}=\left(u_{n}, u_{n+1}, u_{n+2}, u_{n+3}\right)$ a sequence in $\mathbb{Z}_{r}^{4}$ formed by consecutive terms of the $u$ sequence read mod $r$. Let us consider the first $r^{4}+1 U_{i}$ 's, $i=0, \ldots, r^{4}$. Since $\mathbb{Z}_{r}^{4}$ has $r^{4}$ elements, by the Pigeonhole Principle, there are integers $i, j$, such that $U_{i}=U_{j}, 0 \leq i<j \leq r^{4}$. Hence, as $\left\{u_{n}\right\}_{n \in \mathbb{Z}^{+}}$can be defined recursively by the previous four terms of the same sequence, we conclude that $\left\{u_{n}\right\}_{n \in \mathbb{Z}^{+}}(\bmod r)$ is eventually periodic with period $p \mid(j-i)$. Since $u_{n}=3 u_{n-2}-u_{n-4}$ is equivalent to $u_{n-4}=3 u_{n-2}-u_{n}$, it follows that $\left\{u_{n}\right\}_{n \in \mathbb{Z}^{+}}(\bmod r)$ has to be periodic with period $p$. Then, $u_{j-i-1} \equiv_{r} u_{-1} \equiv_{r} 0$ and $\left\{q \in \mathbb{Z}^{+}|r| u_{q-1}\right\} \ni(j-i)$ is non-empty, ending the proof.

Proposition 2.11 $r \mid u_{m-1}$ if and only if $\psi(r) \mid m$. 
Proof: Upon extending $u_{n}$ to the negative integers using the recurrence relation $u_{n-4}=3 u_{n-2}-u_{n}$, we note that $u_{n}=-u_{-n-2}$.

We start by verifying that $u_{n}=-u_{-n-2}$. This is done using the fact that $u_{1}=-u_{-3}=1, u_{0}=$ $-u_{-2}=1$ and $u_{-1}=-u_{-1}=0$. After this, we leave it as an exercise to use induction on $n$ to obtain the desired conclusion.

Claim 2.1 If $u_{\psi(n)-1} \mid u_{l-1}$ then $u_{\psi(n)-1} \mid u_{l \pm \psi(n)-1}$.

Proof: If $\psi(n)$ is even or $(l-1)$ is odd, by Proposition 2.9 we have:

$$
u_{ \pm \psi(n)+(l-1)}=u_{( \pm \psi(n)+1)} u_{l-1}-u_{ \pm \psi(n)-1} u_{l-3}=u_{( \pm \psi(n)+1)} u_{l-1} \mp u_{\psi(n)-1} u_{l-3} .
$$

Otherwise, if $\psi(n)$ is odd and $(l-1)$ is even, by Proposition 2.9 we have:

$$
u_{(l-1) \pm \psi(n)}=u_{(l-1)} u_{ \pm \psi(n)}-u_{l-2} u_{ \pm \psi(n)-1}=u_{(l-1)} u_{ \pm \psi(n)} \mp u_{l-2} u_{\psi(n)-1} .
$$

Either way, if $u_{\psi(n)-1} \mid u_{l-1}$, then $u_{\psi(n)-1} \mid u_{l \pm \psi(n)-1}$. This concludes the proof of the Claim.

Resuming the proof of Proposition 2.11. Claim 2.1 implies, by setting $n=r, m=\psi(r)$ and using induction, that $u_{\psi(r)-1} \mid u_{k \psi(r)-1}$, for any $k \in \mathbb{Z}$. Since $r \mid u_{\psi(r)-1}$, by definition, we have thus proved that if $\psi(r) \mid m$ then $r \mid u_{m-1}$.

Now assume $r \mid u_{m-1}$ and let $R$ be the remainder of the division of $m$ by $\psi(r)$. Then there exists $q \in \mathbb{Z}$ such that $r\left|u_{q \psi(r)+R-1}\right| u_{(q-1) \psi(r)+R-1}|\cdots| u_{R-1}$ invoking the Claim 2.1 in the last passages. Hence, by definition of $\psi(r)$, we get $R=0$, concluding the proof of the Proposition.

\subsubsection{The proof of Theorem 1.2}

The proof of Theorem 1.2 now follows easily thanks to Theorem 2.1 below. We refer the reader to Satoh (2009) and Oshiro (2010) for statement 3., and to Lopes and Matias (2012) for statement 4. We remark that, given two positive integers $a, b$, we let $\langle a, b\rangle$ stand for 1 if $a$ and $b$ are relatively prime, otherwise, we let $\langle a, b\rangle$ stand for their least common prime factor.

Theorem 2.1 Let $r$ be an integer greater than 1.

Let $L$ be a non-split link.

1. $\langle r, \operatorname{det} L\rangle=2$ if, and only if, $\operatorname{mincol}_{r} L=2$,

2. $\langle r, \operatorname{det} L\rangle=3 \quad$ if, and only if, mincol $_{r} L=3$.

Furthermore, if $\operatorname{det} L \neq 0$ then:

3. $\langle r, \operatorname{det} L\rangle \in\{5,7\} \quad$ if, and only if, mincol $_{r} L=4$,

4. $\langle r, \operatorname{det} L\rangle>7, \quad$ if, and only if, mincol $_{r} L \geq 5$. 
Proof: (of Theorem 1.2

1. Knowing that $\psi(2)=3$,

$$
\begin{aligned}
& 2 \mid r \text { and } 3|n \quad \Leftrightarrow \quad 2| r \text { and } \psi(2)|n \quad \Leftrightarrow \quad 2| r \text { and } 2 \mid u_{n-1} \quad \Leftrightarrow \\
& 2 \mid r \text { and } 2 \mid \operatorname{det} T H K(3, n) \quad \Leftrightarrow \quad\langle r, \operatorname{det} T H K(3, n)\rangle=2 \quad \Leftrightarrow \\
& \text { mincol }_{r} T H K(3, n)=2 .
\end{aligned}
$$

2. Knowing that $2 \nmid r$ or $3 \nmid n$ and $\psi(3)=4$,

$$
\begin{aligned}
& 3 \mid r \text { and } 4|n \Leftrightarrow \quad \Leftrightarrow \quad 3| r \text { and } \psi(3)|n \quad \Leftrightarrow \quad 3| r \text { and } 3 \mid u_{n-1} \quad \Leftrightarrow \\
& 3 \mid r \text { and } 3|\operatorname{det} T H K(3, n) \Leftrightarrow \quad \Leftrightarrow r, \operatorname{det} T H K(3, n)\rangle=3 \quad \Leftrightarrow \\
& \text { mincol }_{r} T H K(3, n)=3 .
\end{aligned}
$$

3. The argument for this instance mimics the ones used in the preceding two instances. We leave the details to the reader.

4. The right-hand side of Figure 5 shows an 11-coloring of $T H K(3, n)$ with 5 colors. Hence, Proposition 2.5. Proposition 2.6 along with the preceding instances, imply that if $11 \mid r$ and $5 \mid n$ (and neither $r$ nor $n$ comply with the preceding instances) then mincol $_{r} T H K(3, n)=5$.

\subsection{Proof of Theorem 1.3}

\subsubsection{Preliminaries}

After experimenting with $r$-colorings of the knots $T H K(3, n)$ 's for small values of $r$ and $n$ we came up with the following examples, displayed in Figures 4,5, and 6 Figures 4, 5, and 6 are to be considered upon closure of the braids therein. We do not depict the closure of these braids in order not to over-burden the figures. Inspection of some cases displayed in Figures 4, 5, and 6 shows we have some control on the colors down the right hand-side of the standard diagrams with respect to the left-hand side. This allows us to decrease the number of colors needed to produce a non-trivial coloring.

Consider the 11-coloring of $T H K(3,5)$ (right-hand side of Figure 5). The sequence of colors down the left-hand side i.e., the $\left(x_{n}\right)$ sequence for $0 \leq n \leq 4$, call it $L$, is

$$
L=(1,2,0,4,7) .
$$

The sequence of colors down the middle i.e., the $\left(y_{n}\right)$ sequence for $0 \leq n \leq 4$, call it $M$, is

$$
M=(7,1,2,0,4) .
$$

Clearly, $M$ is a circular shift of $L$, since $y_{i+1}=x_{i}$ for $i=0,1,2,3,4$, mod 5 . This is a consequence of the arrangement of the arcs in any standard diagram of $T H K(3, n)$. Hence, the equality of the sequences $L$ and $M$ modulo circular shift is true for any such diagram. 

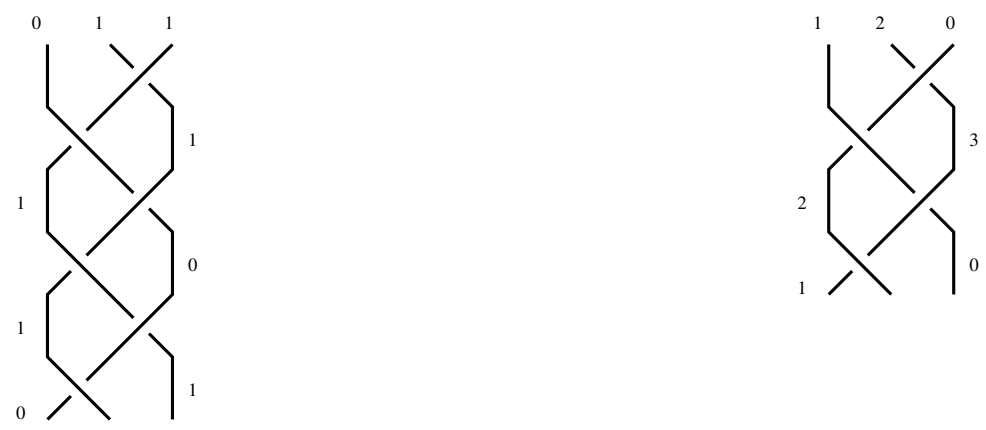

Fig. 4: Illustrative examples of non-trivial colorings of $T H K(3, n)$ 's. A 2-coloring of $T H K(3,3)$ with 2 colors and a 5-coloring of $T H K(3,2)$ with 4 colors.
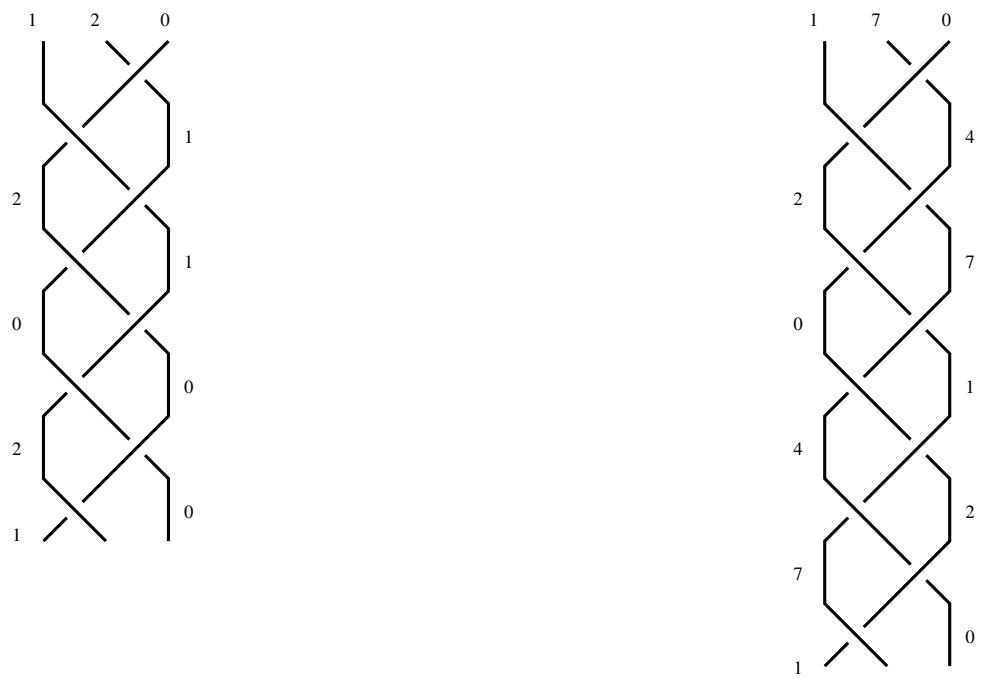

Fig. 5: Illustrative examples of non-trivial colorings of $T H K(3, n)$ 's (cont'd). A 3 -coloring of $T H K(3,4)$ with 3 colors and an 11-coloring of $T H K(3,5)$ with 5 colors. 


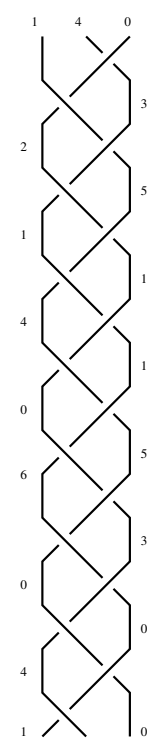

Fig. 6: Illustrative examples of non-trivial colorings of $T H K(3, n)$ 's (end). A 7-coloring of $T H K(3,8)$ with 7 colors.

Let us consider now the sequence of colors down the right-hand side i.e., $\left(z_{n}\right)$ for $0 \leq n \leq 4$, call it $R$, of the 11-coloring of $\operatorname{THK}(3,5)$ (right-hand side of Figure 5):

$$
R=(0,4,7,1,2)
$$

Then $R$ is a circular shift of $L$ but now this is not a general feature of non-trivial $r$-colorings on standard diagrams of $T H K(3, n)$ 's (see, for instance, the 5-coloring of $T H K(3,2)$ on the right-hand side of Figure 4. or the 7-coloring of $\operatorname{THK}(3,8)$ in Figure 6).

Bearing in mind that if $\operatorname{gcd}\left(r, u_{n-1}\right)>1$, there is a non-trivial $r$-coloring of $T H K(3, n)$ (Corollary 1.2 , then, provided $r$ is prime, $\psi(r)$ yields the least number of $\sigma_{2} \sigma_{1}^{-1}$ 's we have to juxtapose in order to obtain a non-trivial $r$-coloring for a Turk's head knot on three strands, namely $T H K(3, \psi(r))$. For prime $r>5$ and odd $\psi(r)$, we construct a non-trivial $r$-coloring on the standard diagram of $T H K(3, \psi(r))$ such that the $R$ sequence is a circular shift of the $L$ sequence (Theorem 1.3 . There are, on average, two arcs per $\sigma_{2} \sigma_{1}^{-1}$ in a standard diagram of $\operatorname{TH} K(3, n)$. When $R$ is a circular shift of $L$, we use only one color per $\sigma_{2} \sigma_{1}^{-1}$, on average i.e., we use only $\psi(r)$ colors. This constitutes a reduction in half on the number of colors with respect to the worst case (different arcs, different colors). Moreover, we show that, for prime $r>5$

$$
\psi(r) \mid(r+1) \quad \text { or } \quad \psi(r) \mid(r-1),
$$

so that, if $\psi(r)$ is odd, then $\psi(r) \leq \frac{r+1}{2}$ or $\psi(r) \leq \frac{r-1}{2}$ (Corollary 2.4, below). This guarantees also that we are using roughly half of the colors available, when $r>5$ is prime and $\psi(r)$ is odd.

On the other hand, for prime $r>5$ with $\psi(r)$ even, we show below (Theorem 1.3) that the input color $(0,1,0)$ induces a coloring whose number of colors is less than $\psi(r)-1$. Here however, it may happen that $\psi(r)=r+1$, so that the estimate equals the total number of colors available. 
We develop these ideas below.

\subsubsection{Further analysis of $u_{n}$}

In order to carry out the ideas expressed above, we need a deeper analysis of the sequence $u_{n}$. This is the goal of the current Subsection.

Proposition 2.12 Let $p \neq 5$ be an odd prime. Then:

$$
\left\{\begin{array}{lll}
p \mid u_{p} & \text { if and only if } & 5^{\frac{p-1}{2}} \equiv-1(\bmod p) \\
p \mid u_{\frac{p-3}{2}} & \text { if and only if } & 5^{\frac{p-1}{2}} \equiv 1(\bmod p) .
\end{array}\right.
$$

Proof: As $u_{n}$ is a sequence taking on integer values we may conclude that the coefficients of $\sqrt{5}$, after applying Binomial Theorem to the expression $\sqrt{8}$, will sum zero. Also, one may see that $p \nmid\left(\begin{array}{c}p+2 \\ j\end{array}\right)$ if and only if $j=0,1,2, p, p+1, p+2$ and $p \nmid\left(\begin{array}{c}p \\ j\end{array}\right)$ if and only if $j=0, p$. By Fermat's Little Theorem we have $5^{p-1} \equiv 1$, which implies $5^{\frac{p-1}{2}} \equiv 1$ or $5^{\frac{p-1}{2}} \equiv-1(\bmod \mathrm{p})$. Therefore, $\bmod p$ :

$$
\begin{aligned}
0 & \equiv u_{p} \equiv \frac{1}{2^{p+2}}\left(2 \sum_{i=0}^{\frac{p+1}{2}}\left(\begin{array}{c}
p+2 \\
2 i
\end{array}\right) 5^{\frac{p+1}{2}-i}-8 \sum_{i=0}^{\frac{p-1}{2}}\left(\begin{array}{c}
p \\
2 i
\end{array}\right) 5^{\frac{p-1}{2}-i}\right) \equiv \\
& \equiv \frac{1}{2^{3}}\left(2 \times 5^{\frac{p+1}{2}}+2 \times \frac{(p+2)(p+1)}{2} \times 5^{\frac{p-1}{2}}+2(p+2)-8 \times 5^{\frac{p-1}{2}}\right) \equiv \\
& \equiv \frac{1}{2^{3}}\left(4 \times\left(5^{\frac{p-1}{2}}+1\right)\right),
\end{aligned}
$$

which is equivalent to $5^{\frac{p-1}{2}} \equiv-1, \bmod p$.

Assume, now, $5^{\frac{p-1}{2}} \equiv 1$, $\bmod p$. Then by Euler's Criterion there exists $\alpha \in \mathbb{Z}$, such that $\alpha^{2} \equiv 5$. As in (8) the coefficients of $\sqrt{5}$ will sum zero, and we obtain, $\bmod p$ :

$$
u_{\frac{p-3}{2}} \equiv \alpha^{-1}\left((1+\alpha)^{\frac{p+1}{2}}-4(-1+\alpha)^{\frac{p-3}{2}}-(1-\alpha)^{\frac{p+1}{2}}+4(-1-\alpha)^{\frac{p-3}{2}}\right) 2^{-\frac{p+1}{2}} .
$$

Multiplying both sides by $2^{\frac{p+1}{2}} \alpha(1+\alpha)^{\frac{p+1}{2}}$, applying Fermat's Little Theorem and noting $p \nmid(\alpha \pm 1)$ for $\alpha^{2} \equiv 5 \neq 1$ :

$$
\begin{aligned}
2^{\frac{p+1}{2}} \alpha & (1+\alpha)^{\frac{p+1}{2}} u_{\frac{p-3}{2}} \equiv \\
& \equiv(1+\alpha)^{p+1}-4(1+\alpha)^{2}\left(-1+\alpha^{2}\right)^{\frac{p-3}{2}}-\left(1-\alpha^{2}\right)^{\frac{p+1}{2}}+4(-1)^{\frac{p-3}{2}}(1+\alpha)^{p-1} \equiv \\
& \equiv(1+\alpha)^{2}-4(1+\alpha)^{2}(-1+5)^{\frac{p-3}{2}}-(1-5)^{\frac{p+1}{2}}+4(-1)^{\frac{p-3}{2}} \equiv \\
& \equiv(1+\alpha)^{2}-(1+\alpha)^{2} 2^{p-1}-(-1)^{\frac{p+1}{2}} 2^{p+1}+4(-1)^{\frac{p-3}{2}} \equiv \\
& \equiv(1+\alpha)^{2}-(1+\alpha)^{2}-4(-1)^{\frac{p-3}{2}}+4(-1)^{\frac{p-3}{2}} \equiv 0,
\end{aligned}
$$

which implies $u_{\frac{p-3}{2}} \equiv 0$, mod $p$, concluding the proof. 
Corollary 2.4 Let $p \neq 5$ be an odd prime. Then, $\bmod p$ :

$$
\left\{\begin{array}{lll}
\psi(p) \mid(p+1) & \text { if and only if } & 5^{\frac{p-1}{2}} \equiv-1 \\
\psi(p) \mid\left(\frac{p-1}{2}\right) & \text { if and only if } & 5^{\frac{p-1}{2}} \equiv 1
\end{array}\right.
$$

In particular, when $\psi(p)$ is odd, then $\psi(p) \leq(p+1) / 2$.

Proof: It is a straightforward application of Propositions 2.11 and 2.12

Proposition 2.13 Let p be a prime greater than 5 .

1. If $\psi(p)$ is odd then mincol $T H K(3, \psi(p)) \leq \psi(p)$,

2. If $\psi(p)$ is even then,

(a) If $4 \mid \psi(p)$, then mincol $_{p} T H K(3, \psi(p)) \leq \psi(p)-1$,

(b) If $4 \nmid \psi(p)$, then mincol $_{p} T H K(3, \psi(p)) \leq \psi(p)-5$.

Proof: Let us first suppose $\psi(p)$ is odd, and set $a=1, c=0$ and $b$ arbitrary, for the moment (Figure 77. We will show that $b$ can be chosen so that the $R$ sequence i.e., the sequence $\left.\left(z_{n}\right)\right|_{0 \leq n \leq \psi(p)}$, is a circular

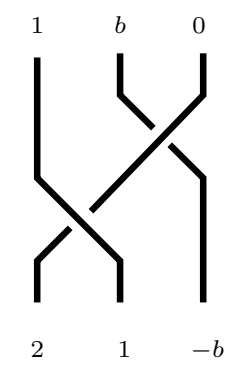

Fig. 7: Propagation of colors down $\sigma_{2} \sigma_{1}^{-1}$ for a particular choice of $a, b, c$.

shift of the $L$ sequence i.e., the sequence $\left.\left(x_{n}\right)\right|_{0 \leq n \leq \psi(p)}$, yielding the required result. As we saw above, in the beginning of this Subsection, this implies that the number of colors is bounded above by $\psi(p)$.

By Proposition 2.1.

$$
x_{k}=3 x_{k-1}-x_{k-2}-1+b \quad \Leftrightarrow \quad\left(x_{k}-1+b\right)=3\left(x_{k-1}-1+b\right)-\left(x_{k-2}-1+b\right) .
$$

and similarly for $y_{k}, z_{k}$. In particular, the above relations tell us that any term of $x_{k}$ (respect., $z_{k}$ ) is obtained from the preceding two terms of the sequence. We then focus on two consecutive terms of the $x$-sequence, $\left(x_{k}, x_{k+1}\right)$ and we will later equate a certain pair of them to $\left(z_{0}, z_{1}\right)=(0,-b)$ in order to obtain the equality between the $R$ and $L$ sequences, modulo circular shift. 
The expressions in (10) lead to:

$$
\begin{aligned}
{\left[\begin{array}{c}
x_{k+1} \\
x_{k}
\end{array}\right] } & =\left[\begin{array}{cc}
3 & -1 \\
1 & 0
\end{array}\right]\left[\begin{array}{c}
x_{k}-1+b \\
x_{k-1}-1+b
\end{array}\right]+\left[\begin{array}{l}
1-b \\
1-b
\end{array}\right]=\left[\begin{array}{cc}
3 & -1 \\
1 & 0
\end{array}\right]\left(\left[\begin{array}{cc}
3 & -1 \\
1 & 0
\end{array}\right]\left[\begin{array}{l}
x_{k-1}-1+b \\
x_{k-2}-1+b
\end{array}\right]\right)+\left[\begin{array}{c}
1-b \\
1-b
\end{array}\right] \\
& =\cdots=\left[\begin{array}{cc}
3 & -1 \\
1 & 0
\end{array}\right]^{k}\left[\begin{array}{l}
x_{1}-1+b \\
x_{0}-1+b
\end{array}\right]+\left[\begin{array}{c}
1-b \\
1-b
\end{array}\right]=\left[\begin{array}{cc}
3 & -1 \\
1 & 0
\end{array}\right]^{k}\left[\begin{array}{c}
1+b \\
b
\end{array}\right]+\left[\begin{array}{c}
1-b \\
1-b
\end{array}\right] .
\end{aligned}
$$

By induction one can prove that:

$$
\left[\begin{array}{cc}
3 & -1 \\
1 & 0
\end{array}\right]^{k}=\left[\begin{array}{ll}
u_{2 k+1} & -u_{2 k-1} \\
u_{2 k-1} & -u_{2 k-3}
\end{array}\right]
$$

and taking determinants on both sides of the preceding equation we obtain:

$$
1=-u_{2 k+1} u_{2 k-3}+u_{2 k-1}^{2} .
$$

In order for $L$ to be a circular shift of $R$ we set $x_{k+1}=z_{1}=-b, x_{k}=z_{0}=0$. Then:

$$
\left[\begin{array}{c}
-b \\
0
\end{array}\right]=\left[\begin{array}{cc}
3 & -1 \\
1 & 0
\end{array}\right]^{k}\left[\begin{array}{c}
1+b \\
b
\end{array}\right]+\left[\begin{array}{c}
1-b \\
1-b
\end{array}\right]
$$

which is equivalent to:

$$
\left[\begin{array}{ll}
u_{2 k+1}+1 & -u_{2 k-1}-1 \\
u_{2 k-1}+1 & -u_{2 k-3}-2
\end{array}\right]\left[\begin{array}{c}
1+b \\
b
\end{array}\right] \equiv_{p}\left[\begin{array}{l}
0 \\
0
\end{array}\right]
$$

The determinant of the coefficient matrix in (11) is given by:

$$
\begin{aligned}
& -u_{2 k+1} u_{2 k-3}+u_{2 k-1}^{2}-2 u_{2 k+1}-u_{2 k-3}+2 u_{2 k-1}-1= \\
& =1+\left(-u_{2 k-3}+3 u_{2 k-1}-u_{2 k+1}\right)-u_{2 k+1}-u_{2 k-1}-1=-\left(u_{2 k+1}+u_{2 k-1}\right)=-u_{2 k} .
\end{aligned}
$$

We then set $k=\frac{\psi(p)-1}{2}$ so that $2 k+1=\psi(p)$. Then $u_{2 k} \equiv 0$, $\bmod p$, by definition of $\psi$, and the kernel associated to the system (11) is non-trivial. If the non-null vectors of this kernel had equal coordinates then in particular,

$$
\left[\begin{array}{l}
0 \\
0
\end{array}\right]=\left[\begin{array}{cc}
u_{\psi(p)}+1 & -u_{\psi(p)-2}-1 \\
* & *
\end{array}\right]\left[\begin{array}{l}
1 \\
1
\end{array}\right]=\left[\begin{array}{c}
u_{\psi(p)}-u_{\psi(p)-2} \\
*
\end{array}\right]=\ldots
$$

and, by Proposition 2.8 as $\psi(p)$ is odd:

$$
\cdots=\left[\begin{array}{c}
u_{\psi(p)}-u_{\psi(p)-2}-\left(u_{\psi(p)}-u_{\psi(p)-1}+u_{\psi(p)-2}\right) \\
*
\end{array}\right]={ }_{p}\left[\begin{array}{c}
-2 u_{\psi(p)-2} \\
*
\end{array}\right] .
$$

Now, $2 u_{\psi(p)-2}={ }_{p} 0$, only if $p=2$ (in which case the proposition is easily verified, as $\psi(2)=3 \geq 2$ ), or $p \mid u_{\psi(p)-2}$, following from Proposition 2.11 that $\psi(p) \mid \psi(p)-1$, which implies $\psi(p)=1$ and $p \mid u_{0}=1$, contradicting the fact that $p$ is prime. We can thus assume that non-trivial elements in the indicated kernel have distinct coordinates, say $(r, s)$ with $r \neq s$ mod $p$.

The vector $\left((r-s)^{-1} r,(r-s)^{-1} s\right)={ }_{p}\left(r^{\prime}, s^{\prime}\right)$ also belongs to this kernel and satisfies $r^{\prime}-s^{\prime}=1$. Therefore $b=s^{\prime}$ is a solution to equation (11). We conclude that, for the top colors $\left(1, s^{\prime}, 0\right)$, the rightmost and leftmost arcs have equal colors in pairs. Therefore, given the recurrence relation satisfied by the colors on each side, the colors used in the leftmost, middle, and rightmost arcs are the same and we use, at most, $\psi(p)$ colors. 
Let us now suppose that $\psi(p)$ is even. By Proposition 2.8 we have:

$$
\frac{u_{\psi(p)}+u_{\psi(p)-2}}{5}=u_{\psi(p)-1}={ }_{p} 0 \Rightarrow u_{\psi(p)}={ }_{p}-u_{\psi(p)-2},
$$

as $p \neq 5$. Remember that $u_{0}=1=-u_{-2}$. So, for some integer $\alpha$ we have $u_{\psi(p)-2}={ }_{p} \alpha u_{0}$ and $u_{\psi(p)}={ }_{p} \alpha u_{-2}$. Furthermore, and as:

$$
u_{k}=3 u_{k-2}-u_{k-4} \quad \Leftrightarrow \quad u_{k-4}=3 u_{k-2}-u_{k},
$$

one can easily see that,

$$
\begin{aligned}
{\left[\begin{array}{c}
u_{-2} \\
u_{0}
\end{array}\right] } & =\left[\begin{array}{cc}
3 & -1 \\
1 & 0
\end{array}\right]\left[\begin{array}{l}
u_{0} \\
u_{2}
\end{array}\right]=\left[\begin{array}{cc}
3 & -1 \\
1 & 0
\end{array}\right]\left[\begin{array}{cc}
3 & -1 \\
1 & 0
\end{array}\right]\left[\begin{array}{l}
u_{2} \\
u_{4}
\end{array}\right]=\cdots=\left[\begin{array}{cc}
3 & -1 \\
1 & 0
\end{array}\right]^{\frac{\psi(p)}{2}}\left[\begin{array}{c}
u_{\psi(p)-2} \\
u_{\psi(p)}
\end{array}\right]= \\
& ={ }_{p} \alpha\left[\begin{array}{cc}
3 & -1 \\
1 & 0
\end{array}\right]^{\frac{\psi(p)}{2}}\left[\begin{array}{c}
u_{0} \\
u_{-2}
\end{array}\right]=\alpha\left[\begin{array}{cc}
3 & -1 \\
1 & 0
\end{array}\right]^{\frac{\psi(p)}{2}-1}\left[\begin{array}{l}
u_{2} \\
u_{0}
\end{array}\right]=\cdots=\alpha\left[\begin{array}{c}
u_{\psi(p)} \\
u_{\psi(p)-2}
\end{array}\right]={ }_{p} \alpha^{2}\left[\begin{array}{c}
u_{-2} \\
u_{0}
\end{array}\right]
\end{aligned}
$$

Then $\alpha^{2}={ }_{p} 1 \Leftrightarrow \alpha={ }_{p} \pm 1$. We will now show that $\alpha \equiv 1, \bmod p$.

Assume to the contrary and suppose $\alpha \equiv-1$. Then $u_{0} \equiv-u_{\psi(p)-2}, u_{2} \equiv 3 u_{0}-u_{-2} \equiv-3 u_{\psi(p)-2}+$ $u_{\psi(p)} \equiv-u_{\psi(p)-4}$ and, by applying Proposition 2.8 twice, we get $u_{1}=1 \equiv-u_{\psi(p)-3}$. Using the recurrence satisfied by $u_{n}$, we obtain,

$$
u_{-2+i} \equiv-u_{\psi(p)-i}, i=0,1, \ldots, \psi(p)+2 .
$$

In particular, $\alpha \equiv-1$ implies $u_{-2+\frac{\psi(p)}{2}+1} \equiv-u_{\psi(p)-\frac{\psi(p)}{2}-1} \Leftrightarrow u_{\frac{\psi(p)}{2}-1} \equiv 0$, which contradicts the definition of $\psi(p)$.

Thus $\alpha=1$ and $u_{\psi(p)-2} \equiv 1 \equiv-u_{\psi(p)}$.

We note that $\left(x_{0}, y_{0}, z_{0}\right)=(0,1,0)$ induces a non-trivial $p$-coloring on $T H K(3, \psi(p))$, since, by definition, $p \mid u_{\psi(p)-1}$. Then, $\left(x_{\psi(p)}, y_{\psi(p)}, z_{\psi(p)}\right)=(0,1,0)$.

Arguing as above in the odd $\psi(p)$ case, we obtain:

$$
\begin{aligned}
& {\left[\begin{array}{c}
x_{k+1} \\
x_{k}
\end{array}\right]=\left[\begin{array}{cc}
3 & -1 \\
1 & 0
\end{array}\right]^{k}\left[\begin{array}{l}
x_{1}+1 \\
x_{0}+1
\end{array}\right]+\left[\begin{array}{l}
-1 \\
-1
\end{array}\right]=\left[\begin{array}{ll}
u_{2 k+1} & -u_{2 k-1} \\
u_{2 k-1} & -u_{2 k-3}
\end{array}\right]\left[\begin{array}{l}
1 \\
1
\end{array}\right]+\left[\begin{array}{l}
-1 \\
-1
\end{array}\right]} \\
& {\left[\begin{array}{c}
z_{k+1} \\
z_{k}
\end{array}\right]=\left[\begin{array}{cc}
3 & -1 \\
1 & 0
\end{array}\right]^{k}\left[\begin{array}{l}
z_{1}+1 \\
z_{0}+1
\end{array}\right]+\left[\begin{array}{l}
-1 \\
-1
\end{array}\right]=\left[\begin{array}{ll}
u_{2 k+1} & -u_{2 k-1} \\
u_{2 k-1} & -u_{2 k-3}
\end{array}\right]\left[\begin{array}{l}
0 \\
1
\end{array}\right]+\left[\begin{array}{l}
-1 \\
-1
\end{array}\right] .}
\end{aligned}
$$

Using these expressions along with $u_{\psi(p)-2} \equiv 1 \equiv-u_{\psi(p)}$ and Proposition 2.8, we obtain the colors displayed in Figure 8 .

By Proposition 2.1, the following hold:

$$
\begin{gathered}
x_{k}=3 x_{k-1}-x_{k-2}+1 \quad \Leftrightarrow \quad x_{k-2}=3 x_{k-1}-x_{k}+1 \\
z_{k}=3 z_{k-1}-z_{k-2}+1 \quad \Leftrightarrow \quad z_{k-2}=3 z_{k-1}-z_{k}+1 .
\end{gathered}
$$

Since $x_{1}=0=x_{\psi(p)}$ and $x_{2}=1=x_{\psi(p)-1}$, then, the sequence $\left.\left(x_{i}\right)\right|_{1 \leq i \leq \psi(p)}$ contains at most $\frac{\psi(p)}{2}$ distinct terms. 

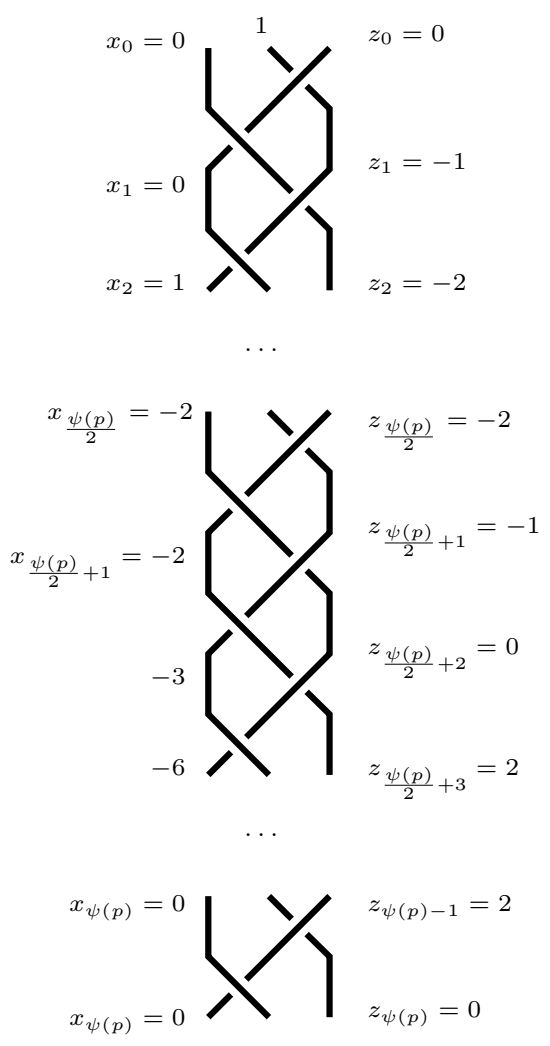

Fig. 8: Minimizing the number of colors for prime $p$ with $\psi(p)$ even.

Assume $4 \mid \psi(p)$ and consider the sequence $\left.\left(z_{i}\right)\right|_{1 \leq i \leq \frac{\psi(p)}{2}+1}$. We have, $z_{1}=-1=z_{\frac{\psi(p)}{2}+1}$ and $z_{2}=-2=z_{\frac{\psi(p)}{2}}$, then there are equal terms in two's. Since $\frac{\psi(p)}{2}+1$ is odd, this means there is a central term, $z_{\frac{\psi(p)}{4}+1}$, and the remaining terms are equal in two's. Thus, this sequence contributes with $\frac{\psi(p)}{4}+1$ distinct terms.

Now for the sequence $\left.\left(z_{i}\right)\right|_{\frac{\psi(p)}{2}+2 \leq i \leq \psi(p)}$. Reasoning as in the preceding case, this sequence has at most, $\frac{\psi(p)-(\psi(p) / 2+2)}{2}+1=\frac{\psi(p)}{4}$.

So, in this $4 \mid \psi(p)$ instance, the number of distinct colors is at most

$$
\frac{\psi(p)}{2}+\left(\frac{\psi(p)}{4}+1\right)+\frac{\psi(p)}{4}-2=\psi(p)-1
$$

where the -2 stems from the fact that 0 and -2 appear on both the $x$ and the $z$ sequences (see Figure 8 ). Assume now $4 \nmid \psi(p)$ and consider the sequence $\left.\left(z_{i}\right)\right|_{1 \leq i \leq \frac{\psi(p)}{2}+1}$. Here $\frac{\psi(p)}{2}+1$ is even, so this sequence is made up of $\frac{\psi(p) / 2+1}{2}$ pairs of equal terms. Moreover, there are two equal consecutive terms, 
$z_{\frac{\psi(p) / 2+1}{2}}=z_{\frac{\psi(p) / 2+3}{2}}$ (see Figure 9, with $c$ the common value). Given the arrangement of the arcs in these standard diagrams, this implies that the $z_{\underline{\psi(p) / 2+1}}^{2} \equiv c$ (see Figure 9p. Using the coloring condition at the crossings and the recurrence relation $z_{k-2}=3 z_{k-1}-z_{k}+1$, we obtain the other colors in Figure 9. which tell us that there are two colors in $\left.\left(z_{i}\right)\right|_{1 \leq i \leq \frac{\psi(p)}{2}+1}$ that already showed up in the $x$ sequence: $c$ and -1 . Thus, the net contribution of $\left.\left(z_{i}\right)\right|_{1 \leq i \leq \frac{\psi(p)}{2}+1}$ is at most $\frac{\psi(p) / 2+1}{2}-2$.

Now, for the sequence $\left.\left(z_{i}\right)\right|_{\frac{\psi(p)}{2}+2 \leq i \leq \psi(p)}$. Again, we have an even number of terms, $\frac{\psi(p)}{2}-1$, equal in two's. Since $z_{\frac{\psi(p)}{2}+2} \equiv \stackrel{2}{0} \equiv z_{\psi(p)}$, which has already been accounted for in the $x$ sequence, then we will consider only $\frac{\frac{\psi(p)}{2}-3}{2}$ pairs of terms. Moreover, there is again a phenomenon analogous to the one depicted in Figure 9, induced by the fact that there are two equal consecutive terms in the $\left.\left(z_{i}\right)\right|_{\frac{\psi(p)}{2}+2 \leq i \leq \psi(p)}$ sequence. This time is just the $c$ that has to be considered. Moreover, we will now prove that this $c$ is distinct from the $c$ occurring in connection with the sequence $\left.\left(z_{i}\right)\right|_{1 \leq i \leq \frac{\psi(p)}{2}+1}$. If they were equal, since the next term in both subsequences, $\left.\left(x_{i}\right)\right|_{1 \leq i \leq \frac{\psi(p)}{2}}$ and $\left.\left(x_{i}\right)\right|_{\frac{\psi(p)}{2}+1 \leq i \leq \psi(p)}$, is -1 $(\bmod p)$, then, given the recurrence relation $z_{k}=3 z_{k-1}-z_{k-2}+1$, then the subsequences would be equal. But they are not equal. Hence the net contribution of $\left.\left(z_{i}\right)\right|_{\frac{\psi(p)}{2}+2 \leq i \leq \psi(p)}$ to the total number of colors is at most $\frac{\frac{\psi(p)}{2}-3}{2}-1$.

Finally, the total number of colors in this $4 \nmid \psi(p)$ instance is at most (recall that -2 shows up on both sides):

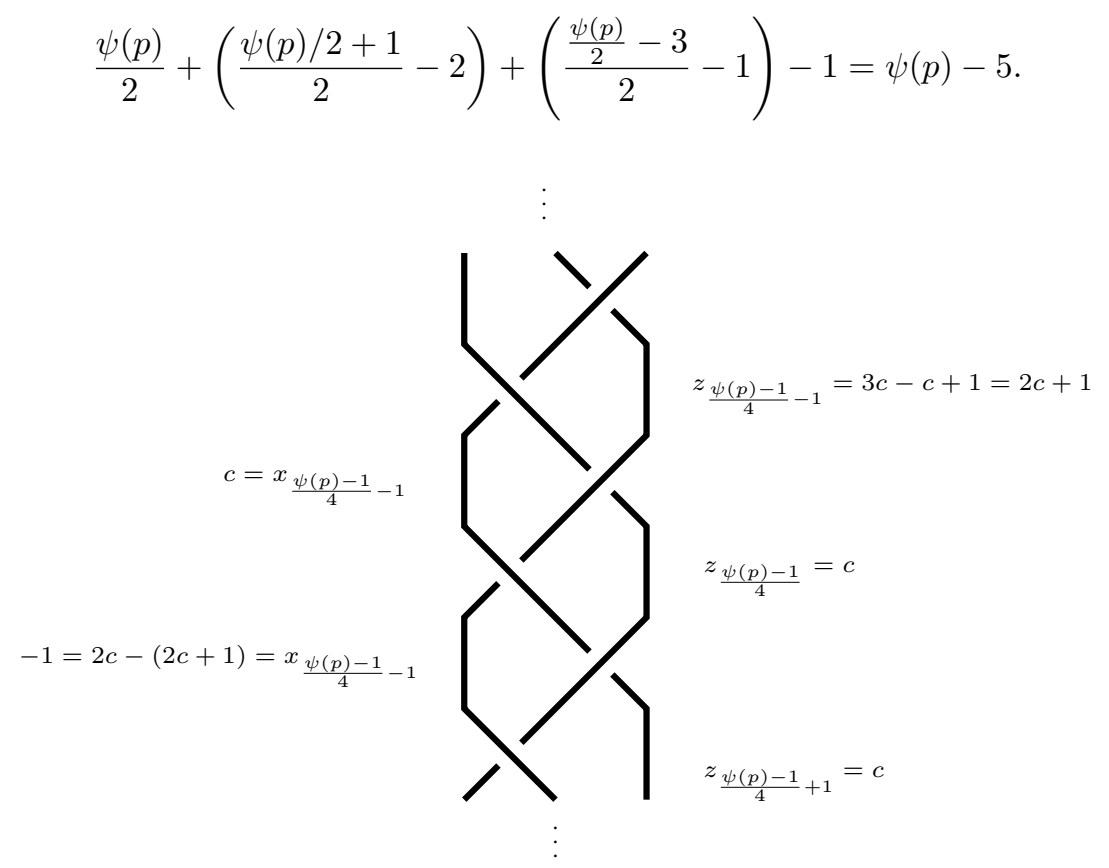

Fig. 9: The colors around $z_{\frac{\psi(p)}{2}+1}, z_{\frac{\psi(p)}{2}+1+1}$ for prime $p$ with $\psi(p)$ even and $4 \nmid \psi(p)$. 
This concludes the proof for the $\psi(p)$ even case, concluding the proof of Proposition 2.12

\subsubsection{Proof of Theorem 1.3 and Corollary 1.3}

The proof of Theorem 1.3 is a straightforward application of Proposition 2.12 .

As for proof of Corollary 1.3 given positive integers $n$ and $r>1$, we choose a common prime factor of $r$ and $u_{n-1}$ (in order to ensure there are non-trivial colorings), which minimizes $\psi$ (in order to minimize the number of colors involved. Applying Propositions 2.6 and 2.5. we conclude the proof of Corollary 1.3 .

\section{Acknowledgements}

P.L. acknowledges support by the Fundação para a Ciência e a Tecnologia (FCT / Portugal). P.L. and J.M. acknowledge support by the Gulbenkian Foundation (Portugal) in connection with the 2008/2009 edition of the programme "Novos Talentos em Matemática".

\section{References}

M. Asaeda, J. Przytycki, and A. Sikora. Kauffman-Harary conjecture holds for Montesinos knots. J. Knot Theory Ramifications, 13(4):467-477, 2004.

J. Birman. Braids, links, and mapping class groups, volume 82 of Annals of Math. Studies. Princeton University Press, Princeton, N. J., 1974.

R. Fox. A quick trip through knot theory. In J. M. K. Fort, editor, Topology of 3-Manifolds and Related Topics, Georgia, pages 120-167. Prentice-Hall, 1961.

F. Harary and L. Kauffman. Knots and graphs I. Arc graphs and colorings. Adv. in Appl. Math., 22(3): 312-337, 1999.

P. Henrici. Elements of numerical analysis. John Wiley \& Sons, Inc., New York-London-Sydney, 1964.

L. Kauffman. Knots and physics, volume 1 of Series on Knots and Everything. World Scientific Publishing Co., River Edge, NJ, 4th edition, 2013.

L. Kauffman and P. Lopes. On the minimum number of colors for knots. Adv. in Appl. Math., 40(1): 36-53, 2008.

L. Kauffman and P. Lopes. Determinants of rational knots. Discrete Math. Theor. Comput. Sci., 11(2): 111-122, 2009.

W. Lickorish. An introduction to knot theory, volume 175 of Graduate Texts in Mathematics. Springer Verlag, New York, 1997.

P. Lopes. Quandles at finite temperatures I. J. Knot Theory Ramifications, 12(2):159-186, 2003. 
P. Lopes and J. Matias. Minimum number of Fox colors for small primes. J. Knot Theory Ramifications, 21(3), 2012.

T. Mattman and P. Solis. A proof of the Kauffman-Harary conjecture. Algebr. Geom. Topol., 9:2027-2039, 2009.

K. Oshiro. Any 7-colorable knot can be colored by four colors. J. Math. Soc. Japan, 62(3):963-973, 2010.

D. Rolfsen. Knots and links. Chelsea. AMS, 1976.

M. Saito. The minimum number of Fox colors and quandle cocycle invariants. J. Knot Theory Ramifications, 19(11):1449-1456, 2010.

S. Satoh. 5-colored knot diagram with four colors. Osaka J. Math., 46(4):939-948, 2009. 


\section{A Table of $\psi$}

\begin{tabular}{|c|c|c|c|c|c|c|c|c|c|}
\hline $\mathbf{r}$ & $\psi(\mathbf{r})$ & $\mathbf{r}$ & $\psi(\mathbf{r})$ & $\mathbf{r}$ & $\psi(\mathbf{r})$ & $r$ & $\psi(\mathbf{r})$ & $r$ & $\psi(\mathbf{r})$ \\
\hline $\bar{~} \overline{c 1}$ & 2 & 38 & 9 & 75 & $\begin{array}{lll}100 \\
\end{array}$ & 1112 & 24 & 149 & $\overline{774}$ \\
\hline$\overline{2}$ & 3 & 39 & 28 & 76 & 9 & 113 & 38 & 150 & 300 \\
\hline 3 & 4 & 40 & 30 & 77 & 40 & 114 & 36 & 151 & 25 \\
\hline 4 & 3 & 41 & 20 & 78 & 84 & 115 & 120 & 152 & 18 \\
\hline 5 & 10 & 42 & 24 & 79 & 39 & 116 & 21 & 153 & 36 \\
\hline 6 & 12 & 43 & 44 & 80 & 60 & 117 & 84 & 154 & 120 \\
\hline 7 & 8 & 44 & 15 & 81 & 108 & 118 & 87 & 155 & 30 \\
\hline 8 & 6 & 45 & 60 & 82 & 60 & 119 & 72 & 156 & 84 \\
\hline 9 & 12 & 46 & 24 & 83 & 84 & 120 & 60 & 157 & 158 \\
\hline 10 & 30 & 47 & 16 & 84 & 24 & 121 & 55 & 158 & 39 \\
\hline 11 & 5 & 48 & 12 & 85 & 90 & 122 & 30 & 159 & 108 \\
\hline 12 & 12 & 49 & 56 & 86 & 132 & 123 & 20 & 160 & 120 \\
\hline 13 & 14 & 50 & 150 & 87 & 28 & 124 & 15 & 161 & 24 \\
\hline 14 & 24 & 51 & 36 & 88 & 30 & 125 & 250 & 162 & 28 \\
\hline 15 & 20 & 52 & 42 & 89 & 22 & 126 & 24 & 163 & 164 \\
\hline 16 & 12 & 53 & 54 & 90 & 60 & 127 & 128 & 164 & 60 \\
\hline 17 & 18 & 54 & 36 & 91 & 56 & 128 & 96 & 165 & 20 \\
\hline 18 & 12 & 55 & 10 & 92 & 24 & 129 & 44 & 166 & 84 \\
\hline 19 & 9 & 56 & 24 & 93 & 60 & 130 & 210 & 167 & 168 \\
\hline 20 & 30 & 57 & 36 & 94 & 48 & 131 & 65 & 168 & 24 \\
\hline 21 & 8 & 58 & 21 & 95 & 90 & 132 & 60 & 169 & 182 \\
\hline 22 & 15 & 59 & 29 & 96 & 24 & 133 & 72 & 170 & 90 \\
\hline 23 & 24 & 60 & 60 & 97 & 98 & 134 & 204 & 171 & 36 \\
\hline 24 & 12 & 61 & 30 & 98 & 168 & 135 & 180 & 172 & 132 \\
\hline 25 & 50 & 62 & 15 & 99 & 60 & 136 & 18 & 173 & 174 \\
\hline 26 & 42 & 63 & 24 & 100 & 150 & 137 & 138 & 174 & 84 \\
\hline 27 & 36 & 64 & 48 & 101 & 25 & 138 & 24 & 175 & 200 \\
\hline 28 & 24 & 65 & 70 & 102 & 36 & 139 & 23 & 176 & 60 \\
\hline 29 & 7 & 66 & 60 & 103 & 104 & 140 & 120 & 177 & 116 \\
\hline 30 & 60 & 67 & 68 & 104 & 42 & 141 & 16 & 178 & 66 \\
\hline 31 & 15 & 68 & 18 & 105 & 40 & 142 & 105 & 179 & 89 \\
\hline 32 & 24 & 69 & 24 & 106 & 54 & 143 & 70 & 180 & 60 \\
\hline 33 & 20 & 70 & 120 & 107 & 36 & 144 & 12 & 181 & 45 \\
\hline 34 & 18 & 71 & 35 & 108 & 36 & 145 & 70 & 182 & 168 \\
\hline 35 & 40 & 72 & 12 & 109 & 54 & 146 & 222 & 183 & 60 \\
\hline 36 & 12 & 73 & 74 & 110 & 30 & 147 & 56 & 184 & 24 \\
\hline 37 & 38 & 74 & 114 & 111 & 76 & 148 & 114 & 185 & 190 \\
\hline
\end{tabular}

Tab. 2: Table of $\psi$. In bold: prime $r$ 's and their $\psi$ 's. 